\title{
On the Boundary Components of Central Streams
}

\author{
Nobuhiro Higuchi
}

\begin{abstract}
Foliations on the space of $p$-divisible groups were studied by Oort in 2004 . In his theory, special leaves called central streams play an important role. In this paper, we give a complete classification of the boundary components of the central streams for an arbitrary Newton polygon in the unpolarized case. Hopefully this classification would help us to know the boundaries of other leaves and more detailed structure of the boundaries of central streams.
\end{abstract}

\section{Introduction}

In [12], Oort defined the notion of leaves on a family of $p$-divisible groups, which are often called Barsotti-Tate groups, to study the moduli space of abelian varieties in positive characteristic. Let $k$ be an algebraically closed field of characteristic $p$. Let $\mathrm{S}$ be a noetherian scheme over $k$. For a $p$-divisible group $Y$ over $k$, Oort introduced in [12, 2.1] a locally closed subset $\mathcal{C}_{Y}(\mathrm{~S})$ for a $p$-divisible group $\mathcal{Y}$ over $\mathrm{S}$ characterized by the condition that $s$ belongs to $\mathcal{C}_{Y}(\mathrm{~S})$ if and only if $\mathcal{Y}_{s}$ is isomorphic to $Y$ over an algebraically closed field containing $k(s)$ and $k$, see the first paragraph of Section 2.1 for a review. If $\mathcal{Y} \rightarrow \mathrm{S}$ is a universal family over a deformation space or a moduli space, then we call $\mathcal{C}_{Y}(\mathrm{~S})$ the central leaf associated to $Y$ and $\mathcal{Y}$.

In $[12,2.2]$ Oort showed that $\mathcal{C}_{Y}(\mathrm{~S})$ is closed in an open Newton polygon stratum. We consider $\mathcal{C}_{Y}(\mathrm{~S})$ as a locally closed subscheme of $\mathrm{S}$ by giving the induced reduced scheme structure. We are interested in the boundaries of leaves on the deformation space, the problem of which will be formulated from the next paragraph. See $[12,6.10]$ for a question in the polarized case (i.e., the case that $p$-divisible groups associated with polarized abelian varieties).

Let us formulate the problem on the boundaries of central leaves. Fix a $p$-divisible group $X_{0}$ over $k$. Let $\mathfrak{D e f}\left(X_{0}\right)=\operatorname{Spf}(\Gamma)$ be the deformation space of $X_{0}$. The deformation space is the formal scheme pro-representing the functor $\operatorname{Art}_{k} \rightarrow$ Sets which sends $R$ to the set of isomorphism classes of $p$-divisible groups $X$ over $R$ such that $X_{k} \simeq X_{0}$. Here $\mathrm{Art}_{k}$ denotes the category of local Artinian rings with residue field $k$. Let $\mathfrak{X}^{\prime} \rightarrow \operatorname{Spf}(\Gamma)$

Received May 28, 2020; Accepted March 7, 2021.

Communicated by Chia-Fu Yu.

2020 Mathematics Subject Classification. Primary: 14L15; Secondary: 14L05, 14K10.

Key words and phrases. p-divisible group, deformation space, Newton polygons. 
be the universal $p$-divisible group. In $[5,2.4 .4]$ de Jong proved that the category of $p$ divisible groups over $\operatorname{Spf}(\Gamma)$ is equivalent to the category of the $p$-divisible groups over $\operatorname{Spec}(\Gamma)=: \operatorname{Def}\left(X_{0}\right)$. Let $\mathfrak{X}$ be the $p$-divisible group over $\operatorname{Def}\left(X_{0}\right)$ obtained from $\mathfrak{X}^{\prime}$ by this equivalence. Oort studied $\mathcal{C}_{X_{0}}\left(\operatorname{Def}\left(X_{0}\right)\right)$, see [12, 2.7]. We are interested in $\mathcal{C}_{Y}(\operatorname{Def}(X))$ for $X \neq Y$ with $\mathcal{Y}=\mathfrak{X}$. Here is a basic problem.

Problem 1.1. Let $Y$ be a $p$-divisible group over $k$. Classify $p$-divisible groups $X$ over $k$ such that $\mathcal{C}_{Y}(\operatorname{Def}(X)) \neq \emptyset$. Here $\mathcal{C}_{Y}(\operatorname{Def}(X)) \neq \emptyset$ means that $X$ appears as a specialization of a family of $p$-divisible groups whose geometric fibers are isomorphic to $Y$.

In this paper we discuss the case that the $p$-divisible group $Y$ is "minimal", as the general case looks difficult. Oort introduced the notion of minimal $p$-divisible groups in [13, 1.1]. Oort showed in [13, 1.2] that the property: Let $X$ be a minimal p-divisible group over $k$, and let $Y$ be a p-divisible group over $k$. If $X[p] \simeq Y[p]$, then $X \simeq Y$, where $X[p]$ is the kernel of $p$-multiplication. For a Newton polygon $\xi$, we obtain the minimal $p$ divisible group $H(\xi)$. See the third and fourth paragraphs of Section 2.1 for the definitions of Newton polygons and minimal $p$-divisible groups.

For $\mathcal{C}_{Y}\left(\operatorname{Def}\left(X_{0}\right)\right)$, if $Y$ is minimal, then we call it a central stream. This notion is a "central" tool in the theory of foliations. For instance, it is known that the difference between central leaves and central streams comes from isogenies of $p$-divisible groups. Thus to study boundaries of general leaves, it is natural to start with investigating boundaries of central streams.

Let $\xi$ be a Newton polygon. For the notation as above, we may treat the problem.

Problem 1.2. Classify $p$-divisible groups $X$ over $k$ such that $\mathcal{C}_{H(\xi)}(\operatorname{Def}(X)) \neq \emptyset$.

Let us translate this problem into the terminology of the Weyl group of $G L_{h}$. Let $W=W_{h}$ be the Weyl group of $G L_{h}$. We identify this $W$ with the symmetric group $\mathfrak{S}_{h}$ in the usual way. We define $J=J_{c}$ by $J_{c}=\left\{s_{1}, \ldots, s_{h}\right\}-\left\{s_{c}\right\}$, where $s_{i}$ is the simple reflection $(i, i+1)$. Put $d=h-c$. Then there exists a one-to-one correspondence between the isomorphism classes of $\mathrm{BT}_{1}$ 's of rank $p^{h}$ and dimension $d$ over $k$ and the subset ${ }^{J} W$ of $W$, see Section 2.2. Let $X$ be a $p$-divisible group. Let $w \in{ }^{J} W$. We say $w$ is the $(p$-kernel $)$ type of $X[p]$ if the $\mathrm{BT}_{1} X[p]$ corresponds to $w$ by this bijection.

In Proposition 2.1 we will show that for $p$-divisible groups $X$ and $Y$ over $k$ with $X[p] \simeq Y[p]$, if $\mathcal{C}_{H(\xi)}(\operatorname{Def}(X)) \neq \emptyset$, then $\mathcal{C}_{H(\xi)}(\operatorname{Def}(Y)) \neq \emptyset$. Thanks to this proposition, Problem 1.2 is reduced to

Problem 1.3. Classify elements $w$ of ${ }^{J} W$ such that

there exists a $p$-divisible group $X$ over $k$ such that $w$ is the type of $X[p]$ and $\mathcal{C}_{H(\xi)}(\operatorname{Def}(X)) \neq \emptyset$. 
As it seems difficult to answer Problem 1.3 generally, we want to formulate a problem which is a little more restricted than this problem. For this, we recall the notion of specializations of $p$-divisible groups. Let $X$ and $Y$ be $p$-divisible groups over $k$. We say $X$ is a specialization of $Y$ if there exists a family of $p$-divisible group $\mathfrak{X} \rightarrow \operatorname{Spec}(R)$ with discrete valuation ring $R$ in characteristic of $p$ such that $\mathfrak{X}$ is isomorphic to $Y$ over an algebraically closed field containing $L$ and $k$, and $\mathfrak{X}_{k}$ is isomorphic to $X$ over an algebraically closed field containing $K$ and $k$, where $L$ is the field of fractions of $R$, and $K=R / \mathfrak{m}$ is the residue field of $R$. Note that $X$ is a specialization of $Y$ if and only if $\mathcal{C}_{Y}(\operatorname{Def}(X)) \neq \emptyset$ holds. For a $p$-divisible group $X$, we define the length $\ell(X[p])$ of the $p$-kernel by the length of the element of the Weyl group which is the type of $X[p]$. It is known that for the $p$-divisible group $X_{0}$, the length $\ell\left(X_{0}[p]\right)$ is equal to the dimension of the locally closed subscheme of $\operatorname{Def}\left(X_{0}\right)$ obtained by giving the induced reduced structure to the subset of $\operatorname{Def}\left(X_{0}\right)$ consisting of points $s \in \operatorname{Def}\left(X_{0}\right)$ such that $\mathfrak{X}_{s}^{\prime}[p]$ is isomorphic to $X_{0}[p]$ over an algebraically closed field, see $[15,6.10]$ and $[8,3.1 .6]$. We say a specialization $X$ of $Y$ is generic if $\ell(X[p])=\ell(Y[p])-1$ holds.

In this paper, we treat the following problem.

Problem 1.4. Classify $w \in{ }^{J} W$ satisfying that $(*)$ and $\ell(w)=\ell(H(\xi)[p])-1$ for arbitrary Newton polygons $\xi$.

We often call $w \in{ }^{J} W$ satisfying $\ell(w)=\ell(H(\xi)[p])-1$ generic specialization. The terminology "generic" is justified for the following reasons. Let $X_{0}$ be a $p$-divisible group. Let $w$ be the type of $X_{0}[p]$. We denote by $\mathcal{S}_{w}\left(\operatorname{Def}\left(X_{0}\right)\right)$ the reduced subscheme of $\operatorname{Def}\left(X_{0}\right)=\operatorname{Spec}(\Gamma)$ consisting of $s$ satisfying that $\mathfrak{X}_{s}[p]$ is the type of $w$. Then $\operatorname{dim} \mathcal{S}_{w}\left(\operatorname{Def}\left(X_{0}\right)\right)=\ell(w)$, see [15, 6.10] and [8, 3.1.6]. This justifies the terminology "generic" for elements $w$ of ${ }^{J} W$.

A complete answer to this problem will be given by combining Theorems 1.5 and 1.6 below. In [3], we solved Problem 1.4 for Newton polygons $\xi$ consisting of two slopes satisfying that one slope is less than $1 / 2$ and the other slope is greater than $1 / 2$. In this paper, our proof reduces Problem 1.4 to the case of [3].

For an arbitrary Newton polygon $\xi$, we denote by $B(\xi)$ the set

$$
B(\xi)=\left\{\text { types of } X_{s}[p] \mid X_{\bar{\eta}}=H(\xi) \text { and } \ell\left(X_{s}[p]\right)=\ell\left(X_{\bar{\eta}}[p]\right)-1 \text { for some } X \rightarrow \mathrm{S}\right\}
$$

where $\mathrm{S}=\operatorname{Spec}(R)$ for a discrete valuation $\operatorname{ring}(R, \mathfrak{m}), s=\operatorname{Spec}(\kappa)$ and $\bar{\eta}=\operatorname{Spec}(\bar{K})$ with $\kappa=R / \mathfrak{m}$ and $K=\operatorname{frac}(R)$. Problem 1.4 asks us to determine the set $B(\xi)$. The first result is

Theorem 1.5. Let $\xi=\sum_{i=1}^{z}\left(m_{i}, n_{i}\right)$ be a Newton polygon. Let $\xi_{i}=\left(m_{i}, n_{i}\right)+\left(m_{i+1}, n_{i+1}\right)$ be the Newton polygon consisting of two adjacent segments of $\xi$ for $i=1, \ldots, z-1$. For 
any $w \in B\left(\xi_{i}\right)$, the direct sum $w_{\zeta^{(i)}} \oplus w$ belongs to $B(\xi)$, where $w_{\zeta^{(i)}}$ is the type of $H\left(\zeta^{(i)}\right)[p]$ with $\zeta^{(i)}=\left(m_{1}, n_{1}\right)+\cdots+\left(m_{i-1}, n_{i-1}\right)+\left(m_{i+2}, n_{i+2}\right)+\cdots+\left(m_{z}, n_{z}\right)$. Moreover, the obtained map

$$
\bigsqcup_{i=1}^{z-1} B\left(\xi_{i}\right) \rightarrow B(\xi)
$$

sending $w$ to $w_{\zeta^{(i)}} \oplus w$ is bijective.

This theorem implies that the determining problem of boundaries of central streams is reduced to the case that the Newton polygon consists of two segments. Moreover, for the two slopes case, we will show the following result.

Theorem 1.6. Let $\xi=\left(m_{1}, n_{1}\right)+\left(m_{2}, n_{2}\right)$ be a Newton polygon satisfying that $n_{1} /\left(m_{1}+\right.$ $\left.n_{1}\right)>n_{2} /\left(m_{2}+n_{2}\right) \geq 1 / 2$. Put $\xi^{\mathrm{C}}=\left(m_{1}, n_{1}-m_{1}\right)+\left(m_{2}, n_{2}-m_{2}\right)$. Then the map sending $w$ to $\left.w\right|_{\left\{1, \ldots, n_{1}+n_{2}\right\}}$ gives a bijection from $B(\xi)$ to $B\left(\xi^{\mathrm{C}}\right)$.

For a Newton polygon $\xi=\left(m_{1}, n_{1}\right)+\left(m_{2}, n_{2}\right)$, we set $\xi^{\mathrm{D}}=\left(n_{2}, m_{2}\right)+\left(n_{1}, m_{1}\right)$. By the duality, it is easy to see that the map sending $w$ to the map $i \mapsto l-w(l-i)$, with $l=m_{1}+n_{1}+m_{2}+n_{2}+1$, gives a bijection from $B(\xi)$ to $B\left(\xi^{\mathrm{D}}\right)$. Using repeatedly this duality and Theorem 1.6, we can reduce Problem 1.4 to the case of [3]. There results give a complete answer to Problem 1.4 .

Let us state next problem. Let $\xi$ and $\zeta$ be Newton polygons. We write $\zeta \prec \xi$ if each point of $\zeta$ is above or on $\xi$. Moreover, we say that $\zeta \prec \xi$ is saturated if there exists no Newton polygon $\eta$ such that $\zeta \supsetneqq \eta \supsetneqq \xi$. We consider next problem.

Problem 1.7. For a Newton polygon $\xi$ and a generic specialization $X$ of $H(\xi)$, show that there exists a Newton polygon $\zeta$ such that

$$
H(\zeta) \text { appears as a specialization of } X \text {, and } \zeta \prec \xi \text { is saturated. }
$$

Moreover, determine this $\zeta$.

If we denote by $\operatorname{np}(X)$ by the Newton polygon of $X$, then as $\zeta \prec \xi$ is saturated, we see that $\mathrm{np}(X)=\zeta$, see [6, 2.3.1] and [12, Theorem 2.2]. See also [3, Lemma 2.2].

We use the notation of the paragraph after Problem 1.2. Let $w_{\xi}$ denote the element of ${ }^{J} W$, which is the $p$-kernel type of $H(\xi)[p]$. We can translate Problem 1.7 to a problem with respect to the terminology of the Weyl group of $G L_{h}$, and we prove the problem.

Theorem 1.8. Let $\xi$ be any Newton polygon. Let $w \in{ }^{J} W$ be a generic specialization of $w_{\xi}$. Then there exists a Newton polygon $\zeta$ such that

(i) $\zeta \prec \xi$ is saturated, and 
(ii) $w_{\zeta} \subset w$,

where we say $w^{\prime} \subset w$ if there exists a discrete valuation ring $R$ of characteristic $p$ such that there exists a finite flat commutative group scheme $G$ over $R$ satisfying that $G_{\bar{\kappa}}$ is a $\mathrm{BT}_{1}$ of the type $w^{\prime}$, and $G_{\bar{L}}$ is a $\mathrm{BT}_{1}$ of the type $w$, where $L$ (resp. $\kappa$ ) is the fractional field of $R$ (resp. is the residue field of $R$ ).

Thanks to Theorem 1.5, the case that the Newton polygon $\xi$ consists of two slopes is essential. In fact, for a Newton polygon $\xi=\sum_{i=1}^{z}\left(m_{i}, n_{i}\right)$, take a generic specialization $w$ of $w_{\xi}$. Then we have the corresponding generic specialization $w^{\prime}$ of $w_{\xi_{i}}$ by the bijection of Theorem 1.5 with $\xi_{i}=\left(m_{i}, n_{i}\right)+\left(m_{i+1}, n_{i+1}\right)$. Let $\zeta^{\prime}$ be the Newton polygon satisfying (i) and (ii) of Theorem 1.8 for $\xi_{i}$ and $w^{\prime}$. Then the required Newton polygon $\zeta$ satisfying (i) and (ii) for $\xi$ and $w$ is obtained by

$$
\zeta=\left(m_{1}, n_{1}\right)+\cdots+\left(m_{i-1}, n_{i-1}\right)+\zeta^{\prime}+\left(m_{i+2}, n_{i+2}\right)+\cdots+\left(m_{z}, n_{z}\right) .
$$

This paper is organized as follows. In Section 2, we recall notions of $p$-divisible groups, Newton polygons and truncated Dieudonné modules of level one. We review the classification of $\mathrm{BT}_{1}$ 's. Moreover, we introduce the definition of arrowed binary sequences which is the main tool to show the main results. In Section 3, we treat central streams corresponding to arbitrary Newton polygons, and we show some properties of arrowed binary sequences and Newton polygons. The goal of the section is to give a proof of Theorem 1.5 . In Section 4, we treat central streams corresponding to Newton polygons consisting of two slopes, and give a proof of Theorem 1.6. In Section 5, we determine the Newton polygon of each generic specialization of the minimal $\mathrm{DM}_{1}$ for any Newton polygon, and solve Problem 1.7 by showing Theorem 1.8 .

\section{Preliminaries}

In this section, first we recall the notions of $p$-divisible groups, leaves and Dieudonné modules. In Section 2.2, we review the definition of truncated Barsotti-Tate groups of level one $\left(\mathrm{BT}_{1}\right)$ and a classification of $\mathrm{BT}_{1}$ 's. Moreover, in Section 2.3 we introduce arrowed binary sequences as a generalization of classifying data ${ }^{J} W$ of $\mathrm{BT}_{1}$ 's, which are the main tool to show the main theorems.

\section{1. $p$-divisible groups and Dieudonné modules}

In this section we fix a prime number $p$. Let $h$ be a non-negative integer. Let $\mathrm{S}$ be a scheme in characteristic $p$. A p-divisible group (Barsotti-Tate group) of height $h$ over $\mathrm{S}$ is an inductive system $\left(G_{v}, i_{v}\right)_{v \geq 1}$, where $G_{v}$ is a finite locally free commutative group 
scheme over $\mathrm{S}$ of order $p^{v h}$, and for every $v$, there exists the exact sequence of commutative group schemes

$$
0 \longrightarrow G_{v} \stackrel{i_{v}}{\longrightarrow} G_{v+1} \stackrel{p^{v}}{\longrightarrow} G_{v+1}
$$

with canonical inclusion $i_{v}$. Let $X=\left(G_{v}, i_{v}\right)$ be a $p$-divisible group over S. Let $T$ be a scheme over $\mathrm{S}$. Then we have the $p$-divisible group $X_{T}$ over $T$, which is defined as $\left(G_{v} \times \times_{\mathrm{S}} T, i_{v} \times \mathrm{id}\right)$. For the case that $T$ is a closed point $s$ over $\mathrm{S}$, we call the $p$-divisible group $X_{s}$ the fiber of $X$ over $s$. Let $k$ be an algebraically closed field of characteristic $p$. Let $Y \rightarrow \operatorname{Spec}(k)$ be a $p$-divisible group, and let $\mathcal{Y} \rightarrow \mathrm{S}$ be a $p$-divisible group. In [12, 2.1] Oort defined a leaf by

$$
\mathcal{C}_{Y}(\mathrm{~S})=\left\{s \in \mathrm{S} \mid \mathcal{Y}_{s} \text { is isomorphic to } Y \text { over an algebraically closed field }\right\}
$$

as a set, and showed that $\mathcal{C}_{Y}(\mathrm{~S})$ is closed in a Newton stratum (cf. [12, 2.2]). We regard $\mathcal{C}_{Y}(\mathrm{~S})$ as a locally closed subscheme of $\mathrm{S}$ by giving the induced reduced structure on it.

Let $K$ be a perfect field of characteristic $p$. Let $W(K)$ denote the ring of Witt-vectors with coefficients in $K$. Let $\sigma$ be the Frobenius over $K$. We denote by the same symbol $\sigma$ the Frobenius over $W(K)$ if no confusion can occur. A Dieudonné module over $K$ is a finite $W(K)$-module $M$ equipped with $\sigma$-linear homomorphism $\mathrm{F}: M \rightarrow M$ and $\sigma^{-1}$-linear homomorphism $\mathrm{V}: M \rightarrow M$ satisfying that $\mathrm{F} \circ \mathrm{V}$ and $\mathrm{V} \circ \mathrm{F}$ equal the multiplication by $p$. For each $p$-divisible group $X$, we have the Dieudonné module $\mathbb{D}(X)$ using the covariant Dieudonné functor. The covariant Dieudonné theory says that the functor $\mathbb{D}$ induces a canonical categorical equivalence between the category of $p$-divisible groups over $K$ and that of Dieudonné modules over $K$ which are free as $W(K)$-modules. In particular, there exists a categorical equivalence from the category of finite commutative group schemes over $K$ to that of Dieudonné modules over $K$ which are of finite length.

Let $\left\{\left(m_{i}, n_{i}\right)\right\}_{i}$ be finite number of pairs of coprime non-negative integers satisfying that $\lambda_{i} \geq \lambda_{j}$ for $i<j$, where $\lambda_{i}=n_{i} /\left(m_{i}+n_{i}\right)$ for each $i$. A Newton polygon is a lower convex polygon in $\mathbb{R}^{2}$, which breaks on integral coordinates and consists of slopes $\lambda_{i}$. We write

$$
\sum_{i}\left(m_{i}, n_{i}\right)
$$

for the Newton polygon. We call each coprime pair $\left(m_{i}, n_{i}\right)$ segment. For a Newton polygon $\xi=\sum_{i}\left(m_{i}, n_{i}\right)$, we define the $p$-divisible group $H(\xi)$ by

$$
H(\xi)=\bigoplus_{i} H_{m_{i}, n_{i}}
$$

where $H_{m, n}$ is the $p$-divisible group over $\mathbb{F}_{p}$ whose Dieudonné module is given by

$$
\mathbb{D}\left(H_{m, n}\right)=\bigoplus_{i=1}^{m+n} W\left(\mathbb{F}_{p}\right) e_{i}
$$


with the operations $\mathrm{F}$ and $\mathrm{V}$ satisfying that $\mathrm{F} e_{i}=e_{i-m}, \mathrm{~V} e_{i}=e_{i-n}$ and $e_{i-(m+n)}=p e_{i}$. Note that $H_{m, n}$ is of dimension $n$, and its Serre-dual is of dimension $m$.

We say a $p$-divisible group $X$ is minimal if $X$ is isomorphic to $H(\xi)$ over an algebraically closed field for a Newton polygon $\xi$. For a $p$-divisible group $X$, the $p$-kernel $X[p]$ is obtained by the kernel of the multiplication by $p$. It is known that the Dieudonné module of $H(\xi)[p]$ makes a truncated Dieudonné module of level one (abbreviated as $\left.\mathrm{DM}_{1}\right) \mathbb{D}(H(\xi)[p])$. A $\mathrm{DM}_{1}$ over $K$ of height $h$ is the triple $(N, \mathrm{~F}, \mathrm{~V})$ consisting of a $K$-vector space $N$ of height $h$, a $\sigma$-linear map $\mathrm{F}$ and a $\sigma^{-1}$-linear map $\mathrm{V}$ from $N$ to itself satisfying that $\operatorname{ker} \mathrm{F}=\mathrm{im} \mathrm{V}$ and $\operatorname{im} \mathrm{F}=\operatorname{ker} \mathrm{V}$.

Let $\xi=\sum_{i}\left(m_{i}, n_{i}\right)$ be a Newton polygon. We denote by $N_{\xi}$ the $\mathrm{DM}_{1}$ associated to the $p$-kernel of $H(\xi)$. Then $N_{\xi}$ is described as

$$
N_{\xi}=\bigoplus N_{m_{i}, n_{i}}
$$

where $N_{m, n}$ is the $\mathrm{DM}_{1}$ corresponding to the $p$-kernel of $H_{m, n}$.

We use the same notation as Section 1. The following proposition would be well-known to the specialists, but as any good reference cannot be found, we have give a proof for the reader's convenience. A proof in the polarized case is given in [11, 12.5].

Proposition 2.1. Let $\xi$ be a Newton polygon. Put $Y=H(\xi)$. Let $X$ and $X^{\prime}$ be $p$ divisible groups over an algebraically closed field of characteristic $p$. If $\mathcal{C}_{Y}(\operatorname{Def}(X)) \neq \emptyset$ and $X[p] \simeq X^{\prime}[p]$, then $\mathcal{C}_{Y}\left(\operatorname{Def}\left(X^{\prime}\right)\right) \neq \emptyset$.

Proof. Let $h$ and $c$ be positive integers such that $X[p]$ is the type of $w \in{ }^{J} W$ with $W=W_{h}$ and $J=J_{c}$. Put $d=h-c$. Let $\mathrm{F}$ (resp. V) denote the $\sigma$-linear map (resp. $\sigma^{-1}$-linear map) of the $\mathrm{DM}_{1} \mathbb{D}(X[p])=\mathbb{D}(X) / p \mathbb{D}(X)$ with $\sigma$ the Frobenius. We take a basis $\bar{z}_{d+1}, \ldots, \bar{z}_{h}$ of the image of $\mathrm{V}$, and choose $\bar{z}_{1}, \ldots, \bar{z}_{d} \in \mathbb{D}(X[p])$ so that $\bar{z}_{1}, \ldots, \bar{z}_{h}$ is a basis of $\mathbb{D}(X[p])$. We choose lifts $z_{1}, \ldots, z_{h}$ of $\bar{z}_{1}, \ldots, \bar{z}_{h}$ to $\mathbb{D}(X)$. Then $\left\{z_{1}, \ldots, z_{h}\right\}$ is a basis of $\mathbb{D}(X)$. We write

$$
\left(\begin{array}{ll}
A & B \\
C & D
\end{array}\right)
$$

for the display of $X$ with respect to the basis $z_{1}, \ldots, z_{h}$, where $A$ is the $d \times d$ matrix, and $D$ is the $(h-d) \times(h-d)$ matrix. See [10] for the construction of the display. Then for the Dieudonné module $\mathbb{D}(X)$ of $X$ equipped with the operations $\mathrm{F}$ and $\mathrm{V}$, we have

$$
\left(\mathrm{F} z_{1}, \ldots, \mathrm{F} z_{h}\right)=\left(z_{1}, \ldots, z_{h}\right)\left(\begin{array}{cc}
A & p B \\
C & p D
\end{array}\right)
$$

and

$$
\left(\mathrm{V} z_{1}, \ldots, \mathrm{V} z_{h}\right)=\left(z_{1}, \ldots, z_{h}\right)\left(\begin{array}{cc}
p \alpha & p \beta \\
\gamma & \delta
\end{array}\right)^{\sigma^{-1}}
$$


where $\left(\begin{array}{ll}\alpha & \beta \\ \gamma & \delta\end{array}\right)$ is the inverse matrix of the display of $X$. The operations $\mathrm{F}$ and $\mathrm{V}$ on $\mathbb{D}(X[p])$ satisfy that

$$
\left(\mathrm{F} \bar{z}_{1}, \ldots, \mathrm{F} \bar{z}_{h}\right)=\left(\bar{z}_{1}, \ldots, \bar{z}_{h}\right)\left(\begin{array}{cc}
\bar{A} & 0 \\
\bar{C} & 0
\end{array}\right)
$$

and

$$
\left(\mathrm{V} \bar{z}_{1}, \ldots, \mathrm{V} \bar{z}_{h}\right)=\left(\bar{z}_{1}, \ldots, \bar{z}_{h}\right)\left(\begin{array}{cc}
0 & 0 \\
\bar{\gamma} & \bar{\delta}
\end{array}\right)^{\sigma^{-1}}
$$

For the $p$-divisible group $\mathfrak{X} \rightarrow \operatorname{Spec}(R)$ corresponding to the universal $p$-divisible group over $\operatorname{Spf}(R)$, the display of $\mathfrak{X}$ induces that

$$
\left(\mathrm{F} \bar{z}_{1}, \ldots, \mathrm{F} \bar{z}_{h}\right)=\left(\bar{z}_{1}, \ldots, \bar{z}_{h}\right)\left(\begin{array}{cc}
\bar{A}+\overline{T C} & 0 \\
\bar{C} & 0
\end{array}\right)
$$

and

$$
\left(\mathrm{V} \bar{z}_{1}, \ldots, \mathrm{V} \bar{z}_{h}\right)=\left(\bar{z}_{1}, \ldots, \bar{z}_{h}\right)\left(\begin{array}{cc}
0 & 0 \\
\bar{\gamma} & -\bar{\gamma} \bar{T}+\bar{\delta}
\end{array}\right)^{\sigma^{-1}}
$$

where $\bar{T}$ is an $(h-n) \times n$ matrix on $R$.

Choose an isomorphism from $X[p]$ to $X^{\prime}[p]$. Let $\bar{e}_{i}$ be the element of $\mathbb{D}\left(X^{\prime}[p]\right)$ corresponding to $\bar{z}_{i} \in \mathbb{D}(X[p])$ via the isomorphism. Then $\left\{\bar{e}_{1}, \ldots, \bar{e}_{h}\right\}$ gives a basis of $\mathbb{D}\left(X^{\prime}[p]\right)=\mathbb{D}\left(X^{\prime}\right) / p \mathbb{D}\left(X^{\prime}\right)$. We have then

$$
\left(\mathrm{F} \bar{e}_{1}, \ldots, \mathrm{F} \bar{e}_{h}\right)=\left(\bar{e}_{1}, \ldots, \bar{e}_{h}\right)\left(\begin{array}{cc}
\bar{A} & 0 \\
\bar{C} & 0
\end{array}\right)
$$

and

$$
\left(\mathrm{V} \bar{e}_{1}, \ldots, \mathrm{V} \bar{e}_{h}\right)=\left(\bar{e}_{1}, \ldots, \bar{e}_{h}\right)\left(\begin{array}{cc}
0 & 0 \\
\bar{\gamma} & \bar{\delta}
\end{array}\right)^{\sigma^{-1}} .
$$

Choose a basis $e_{1}, \ldots, e_{h}$ of $D(X)$ which are lifts of $\bar{e}_{i}$. Let

$$
\left(\begin{array}{ll}
a & b \\
c & d
\end{array}\right)
$$

be the display of $X$ with respect to $e_{1}, \ldots, e_{h}$. Note that $\bar{a}=\bar{A}$ and $\bar{c}=\bar{C}$. Let $\mathcal{Y}$ be the $p$-divisible group having

$$
\left(\begin{array}{ll}
1 & T \\
0 & 1
\end{array}\right)\left(\begin{array}{ll}
a & b \\
c & d
\end{array}\right)
$$


as its display, where $T$ is a matrix with $T \bmod p=\bar{T}$. Then for the display of $\mathcal{Y}[p]$, we see

$$
\left(\mathrm{F} \bar{e}_{1}, \ldots, \mathrm{F} \bar{e}_{h}\right)=\left(\bar{e}_{1}, \ldots, \bar{e}_{h}\right)\left(\begin{array}{cc}
\bar{a}+\bar{T} \bar{c} & 0 \\
\bar{c} & 0
\end{array}\right)
$$

and

$$
\left(\mathrm{V} \bar{e}_{1}, \ldots, \mathrm{V} \bar{e}_{h}\right)=\left(\bar{e}_{1}, \ldots, \bar{e}_{h}\right)\left(\begin{array}{cc}
0 & 0 \\
\bar{\gamma} & -\bar{\gamma} \bar{T}+\bar{\delta}
\end{array}\right)^{\sigma^{-1}},
$$

whence $\mathcal{Y}$ belongs to $\mathcal{C}_{Y}\left(\operatorname{Def}\left(X^{\prime}\right)\right)$.

\subsection{Classification of $\mathrm{BT}_{1}$ 's}

In this section, we work over an algebraically closed field $k$. Let us review the classification of truncated Barsotti-Tate groups of level one.

Definition 2.2. A truncated Barsotti-Tate group of level one $\left(\mathrm{BT}_{1}\right)$ is a commutative, finite and flat group scheme $N$ over a scheme in characteristic $p$ satisfying properties $[p]_{N}=0$, and

$$
\begin{aligned}
& \operatorname{im}\left(\mathrm{V}: N^{(p)} \rightarrow N\right)=\operatorname{ker}\left(\mathrm{F}: N \rightarrow N^{(p)}\right), \\
& \operatorname{im}\left(\mathrm{F}: N \rightarrow N^{(p)}\right)=\operatorname{ker}\left(\mathrm{V}: N^{(p)} \rightarrow N\right) .
\end{aligned}
$$

A $\mathrm{DM}_{1}$ appears as a Dieudonné module of a $\mathrm{BT}_{1}$. Let $W=W_{h}$ be the Weyl group of the general linear group $G L_{h}$. This $W$ can be identified with the symmetric group $\mathfrak{S}_{h}$. We denote by $s_{i}$ the simple reflection $(i, i+1)$ in $\mathfrak{S}_{h}=W$. Set $\Omega=\left\{s_{1}, \ldots, s_{h-1}\right\}$. We define $J=J_{c}$ by $J_{c}=\Omega-\left\{s_{c}\right\}$. Put $d=h-c$. For the set $W_{J}:=W_{c} \times W_{d}$, let ${ }^{J} W$ be the set consisting of elements $w$ of $W_{h}$ such that $w$ is the shortest element of ${ }^{J} W \cdot w$, see [1, Chap. IV, Ex. $\S 1(3)]$. Then we have

Theorem 2.3. There exists a one-to-one correspondence

$$
{ }^{J} W \longleftrightarrow\left\{\mathrm{BT}_{1} \text { 's over } k \text { of height } h \text { of dimension } d\right\} / \cong .
$$

Moreover, running over all d, we have

$$
\bigsqcup_{d}^{J} W \longleftrightarrow\{0,1\}^{h}
$$

Kraft [7], Oort [11] and Moonen-Wedhorn [9] show that there exists a one-to-one correspondence

$$
\{0,1\}^{h} \longleftrightarrow\left\{\mathrm{DM}_{1} \text { 's over } k \text { of height } h\right\} / \cong
$$


For $\nu \in\{0,1\}^{h}$, we construct the $\mathrm{DM}_{1} D(\nu)$ as follows. We write $\nu(i)$ for the $i$-th coordinate of $\nu$. Put $N=k e_{1} \oplus \cdots \oplus k e_{h}$. We define maps $\mathrm{F}$ and $\mathrm{V}$ as follows:

$$
\mathrm{F} e_{i}= \begin{cases}e_{j} & j=\#\{l \mid \nu(l)=0, l \leq i\} \text { for } \nu(i)=0 \\ 0 & \text { otherwise }\end{cases}
$$

Let $j_{1}, \ldots, j_{c}$, with $j_{1}<\cdots<j_{c}$, be the natural numbers satisfying $\nu\left(j_{l}\right)=1$. Put $d=h-c$. Then a map $\mathrm{V}$ is defined by

$$
\mathrm{V} e_{i}= \begin{cases}e_{j_{l}} & l=i-d \text { for } i>d \\ 0 & \text { otherwise }\end{cases}
$$

Therefore $D(\nu)$ is given by $D(\nu)=(N, \mathrm{~F}, \mathrm{~V})$. Thus we can identify $\mathrm{DM}_{1}$ 's with sequences consisting of 0 and 1 .

For $w \in{ }^{J} W$, we define $\nu(j)=0$ if and only if $w(j)>c$ for $j=1, \ldots, h$, and we obtain the element $(\nu(1), \nu(2), \ldots, \nu(h))$ of $\{0,1\}^{h}$. This gives a one-to-one correspondence between ${ }^{J} W$ and the subset of $\{0,1\}^{h}$ consisting of elements $\nu$ satisfying $\#\{j \mid \nu(j)=$ $0\}=d$. Thus we obtain a bijection between ${ }^{J} W$ and the set of isomorphism class of $\mathrm{DM}_{1}$ 's over $k$ of height $h$ and dimension $d$.

We say that $w^{\prime}$ is a specialization of $w$, denoted by $w^{\prime} \subset w$, if there exists a discrete valuation ring $R$ of characteristic $p$ such that there exists a finite flat commutative group scheme $G$ over $R$ satisfying that $G_{\bar{\kappa}}$ is a $\mathrm{BT}_{1}$ of the type $w^{\prime}$, and $G_{\bar{L}}$ is a $\mathrm{BT}_{1}$ of the type $w$, where $L$ (resp. $\kappa$ ) is the fractional field of $R$ (resp. is the residue field of $R$ ). A generic specialization $w^{\prime}$ of $w$ is a specialization of $w$ satisfying $\ell\left(w^{\prime}\right)=\ell(w)-1$. Here, we show a lemma used for the construction of generic specializations. We define $x \in W$ by $x(i)=i+d$ if $i \leq c$ and $x(i)=i-c$ otherwise. Let $\theta$ be the map from $W$ to itself defined by $\theta(u)=x u x^{-1}$. By $\left.14,4.10\right]$, we have $w^{\prime} \subset w$ if and only if there exists $u \in W_{J}$ such that $u^{-1} w^{\prime} \theta(u) \leq w$ with the Bruhat order $\leq$. Let us recall [3, Lemma 2.7].

Lemma 2.4. Let $w \in{ }^{J} W$. Let $w^{\prime}$ be a specialization of $w$. If $w^{\prime}$ is generic, then there exist $v \in W$ and $u \in W_{J}$ such that

(i) $v=w s$ for a transposition $s$,

(ii) $\ell(v)=\ell(w)-1$,

(iii) $w^{\prime}=u v \theta\left(u^{-1}\right)$.

\subsection{Arrowed binary sequences}

In order to classify the types of generic specializations of $H(\xi)$, we introduce a new notion arrowed binary sequence which is slightly more generalized than the notion of binary 
sequences $\{0,1\}^{h}$. This notion of arrowed binary sequences is useful for classifying generic specializations of minimal $p$-divisible groups.

Definition 2.5. An arrowed binary sequence (we often abbreviate as ABS) $S$ is the triple $(T, \Delta, \Pi)$ consisting of an ordered symbol set $T=\left\{t_{1}<t_{2}<\cdots<t_{h}\right\}$, a map $\Delta: T \rightarrow\{0,1\}$ and a bijection $\Pi: T \rightarrow T$. For an ABS $S$, let $T(S)$ denote the ordered symbol set of $S$. Similarly, we denote by $\Delta(S)(\operatorname{resp} . \Pi(S))$ the map from $T(S)$ to $\{0,1\}$ (resp. the map from $T(S)$ to itself). For an $\operatorname{ABS} S$, we define the length $\ell(S)$ of $S$ by

$$
\ell(S)=\#\left\{\left(t, t^{\prime}\right) \in T(S) \times T(S) \mid t<t^{\prime} \text { with } \Delta(S)(t)=0 \text { and } \Delta(S)\left(t^{\prime}\right)=1\right\} .
$$

Remark 2.6. Let $N=(N, \mathrm{~F}, \mathrm{~V})$ be a $\mathrm{DM}_{1}$. We construct the arrowed binary sequence $(\Lambda, \delta, \pi)$ associated to $N$ as follows. Let $\nu$ be the element of $\{0,1\}^{h}$ corresponding to $N$. For an totally ordered set $\Lambda=\left\{t_{1}, \ldots, t_{h}\right\}$, let $\delta: \Lambda \rightarrow\{0,1\}$ be the map which sends $t_{i}$ to the $i$-th coordinate of $\nu$. We define a map $\pi: \Lambda \rightarrow \Lambda$ by $\pi\left(t_{i}\right)=t_{j}$, where $j$ is uniquely determined by

$$
\begin{cases}\mathrm{F} e_{i}=e_{j} & \text { if } \delta\left(t_{i}\right)=0 \\ \mathrm{~V} e_{j}=e_{i} & \text { otherwise }\end{cases}
$$

We say an ABS $S$ is admissible if there exists a $\mathrm{DM}_{1}$ such that $S$ is obtained from this $\mathrm{DM}_{1}$ as above.

Remark 2.7. For the $\mathrm{DM}_{1} N_{m, n}$ corresponding to the $p$-divisible group $H_{m, n}$, we get the ABS $S$ as follows. Set $T(S)=\left\{t_{1}<\cdots<t_{m+n}\right\}$. The map $\Delta(S)$ is defined by $\Delta(S)\left(t_{i}\right)=$ 1 if $i \leq m$, and $\Delta(S)\left(t_{i}\right)=0$ otherwise. The map $\Pi(S)$ is defined by $\Pi(S)\left(t_{i}\right)=t_{i+n}$ if $i \leq m$ and $\Pi(S)\left(t_{i}\right)=t_{i-m}$ otherwise.

Let $S$ be an ABS. Put $\delta=\Delta(S)$ and $\pi=\Pi(S)$. The binary expansion $b(t)$ of $t \in T(S)$ is the real number $b(t)=0 . b_{1} b_{2} \ldots$, where $b_{i}=\delta\left(\pi^{-i}(t)\right)$.

Proposition 2.8. Let $S$ be an admissible $A B S$. For elements $t_{i}$ and $t_{j}$ of $T(S)=\left\{t_{1}<\right.$ $\left.t_{2}<\cdots<t_{h}\right\}$, the following holds.

(i) Suppose $\Delta(S)\left(t_{i}\right)=\Delta(S)\left(t_{j}\right)$. Then $t_{i}<t_{j}$ if and only if $\Pi(S)\left(t_{i}\right)<\Pi(S)\left(t_{j}\right)$.

(ii) Suppose $b\left(t_{i}\right) \neq b\left(t_{j}\right)$. Then $b\left(t_{i}\right)<b\left(t_{j}\right)$ if and only if $i<j$.

Proof. (i) follows from the construction of admissible ABS's. Let us see (ii). Put $\delta=\Delta(S)$ and $\pi=\Pi(S)$. By the construction of admissible ABS's, for elements $t$ and $t^{\prime}$ of $T(S)$, if $\delta(t)=1$ and $\delta\left(t^{\prime}\right)=0$, then $\pi\left(t^{\prime}\right)<\pi(t)$. First, assume $b\left(t_{i}\right)<b\left(t_{j}\right)$. Then there exists a non-negative integer $u$ such that $\delta\left(\pi^{-v}\left(t_{i}\right)\right)=\delta\left(\pi^{-v}\left(t_{j}\right)\right)$ for non-negative integers $v$ with $v<u$ and $\delta\left(\pi^{-u}\left(t_{i}\right)\right)=0, \delta\left(\pi^{-u}\left(t_{j}\right)\right)=1$. We have then $\pi^{-u+1}\left(t_{i}\right)<\pi^{-u+1}\left(t_{j}\right)$, and the assertion follows from (i). Next, assume $i<j$. To lead a contradiction, we suppose 
that $b\left(t_{j}\right)<b\left(t_{i}\right)$. Then there exists a non-negative integer $u$ such that $\delta\left(\pi^{-u}\left(t_{j}\right)=0\right.$ and $\delta\left(\pi^{-u}\left(t_{i}\right)\right)=1$, and for non-negative integers $v$ satisfying $v<u$, we have $\delta\left(\pi^{-v}\left(t_{j}\right)=\right.$ $\delta\left(\pi^{-v}\left(t_{i}\right)\right)$. This implies that $\pi^{-u+1}\left(t_{j}\right)<\pi^{-u+1}\left(t_{i}\right)$. We have then $t_{j}<t_{i}$ with $i<j$. By (i), this is a contradiction.

We denote by $\mathcal{H}^{\prime}(h, d)$ the set of admissible ABS's whose corresponding $\mathrm{DM}_{1}$ 's are of height $h$ and dimension $d$. We shall translate the ordering $\subset$ on ${ }^{J} W$ via the bijection from ${ }^{J} W$ to $\mathcal{H}^{\prime}(h, d)$, and we obtain an ordering on $\mathcal{H}^{\prime}(h, d)$ as the notion of specializations of admissible ABS's.

We shall give a method to construct a type of the specializations of ABS's. It will turns out to correspond to specializations $w^{\prime} \subset w$ with $v=w s<w$ and $w^{\prime}=u v \theta\left(u^{-1}\right)$, where $s$ denotes a transposition and $u \in W_{J}$. From $S \in \mathcal{H}^{\prime}(h, d)$, we construct a new admissible ABS $S^{\prime}$.

Definition 2.9. Let $S$ be an ABS with $T(S)=\left\{t_{1}<\cdots<t_{h}\right\}$. Let $i$ and $j$ be natural numbers satisfying that $\Delta(S)\left(t_{i}\right)=0$ and $\Delta(S)\left(t_{j}\right)=1$ with $i<j$. We define an ABS $S^{(0)}$ as follows. We set $T\left(S^{(0)}\right)=\left\{t_{1}^{\prime}<^{\prime} \cdots<^{\prime} t_{h}^{\prime}\right\}$ to be $t_{z}^{\prime}=t_{f(z)}$ for $f=(i, j)$ transposition. Let $\Delta\left(S^{(0)}\right)=\Delta(S)$. For a natural number $z$ with $z \leq h$, we denote by $g(z)$ the natural number satisfying $\Pi(S)\left(t_{z}\right)=t_{g(z)}$. We define $\Pi\left(S^{(0)}\right): T\left(S^{(0)}\right) \rightarrow T\left(S^{(0)}\right)$ by

$$
\Pi\left(S^{(0)}\right)\left(t_{z}^{\prime}\right)= \begin{cases}t_{g(j)}^{\prime} & \text { if } z=i \\ t_{g(i)}^{\prime} & \text { if } z=j \\ t_{g(z)}^{\prime} & \text { otherwise }\end{cases}
$$

Thus we obtain an ABS $S^{(0)}$. We call this ABS small modification by $\left(t_{i}, t_{j}\right)$.

Definition 2.10. Let $S$ be an ABS. Let $S^{(0)}$ be the small modification by $\left(t_{i}, t_{j}\right)$. Put $T\left(S^{(0)}\right)=\left\{t_{1}<\cdots<t_{h}\right\}$. We define an ABS $S^{\prime}$ as follows. Let $T\left(S^{\prime}\right)=T\left(S^{(0)}\right)$ as sets. Let $<^{\prime}$ denote an ordering of $T\left(S^{\prime}\right)$. Put $\Delta\left(S^{\prime}\right)=\Delta\left(S^{(0)}\right)$ and $\Pi\left(S^{\prime}\right)=\Pi\left(S^{(0)}\right)$. We say that the ABS $S^{\prime}$ is a specialization of $S$ by $\left(t_{i}, t_{j}\right)$ if for elements $t_{x}$ and $t_{y}$ of $T\left(S^{\prime}\right)$,

$$
t_{x}<^{\prime} t_{y} \Longrightarrow b\left(t_{x}\right) \leq b\left(t_{y}\right)
$$

We say a specialization $S^{\prime}$ of $S$ is generic if $\ell\left(S^{\prime}\right)=\ell(S)-1$.

Note that for the small modification $S^{(0)}$, in general the specialization $S^{\prime}$ is not unique. However, the $\mathrm{DM}_{1}$ obtained by the pair $\left(T\left(S^{\prime}\right), \Delta\left(S^{\prime}\right)\right)$ is unique. See Example 2.16 .

Remark 2.11. Let $S \in \mathcal{H}^{\prime}(h, d)$. Let $S^{\prime}$ be the specialization of $S$ obtained by exchanging $\left(t_{i}, t_{j}\right)$ with $T\left(S^{\prime}\right)=\left\{t_{1}^{\prime}<^{\prime} \cdots<^{\prime} t_{h}^{\prime}\right\}$. We denote by $w$ the element of ${ }^{J} W$ corresponding to $S$. Put $s=(i, j)$ transposition. Maps $\Pi(S)$ and $\Pi\left(S^{\prime}\right)$ can be regarded as elements of 
$W$. We have then $\Pi(S)=x w$. For the small modification $S^{(0)}$ with $T\left(S^{(0)}\right)=\left\{t_{1}^{(0)}<\right.$ $\left.\cdots<t_{h}^{(0)}\right\}$, we define $\varepsilon \in W$ to be $t_{z}^{(0)}=t_{\varepsilon(z)}^{\prime}$. Since $b\left(t_{z}^{(0)}\right)<0.1$ if $z \leq d$ and $b\left(t_{z}^{(0)}\right)>0.1$ otherwise, $\varepsilon$ stabilizes $\{1,2, \ldots, d\}$. Put $v=w s$. Then $w^{\prime}=u v \theta\left(u^{-1}\right)$ corresponds to $S^{\prime}$ for $u=x^{-1} \varepsilon^{-1} x \in W_{J}$. The map $\Pi\left(S^{\prime}\right)$ is obtained by $\varepsilon^{-1} \Pi(S) s \varepsilon$.

Next, in Definition 2.12 below, we introduce the direct sum of admissible ABS's. The construction of the direct sum is induced from the direct sum of corresponding $\mathrm{DM}_{1}$ 's.

Definition 2.12. Let $S_{1}$ and $S_{2}$ be admissible ABS's. We define the direct sum $S=$ $S_{1} \oplus S_{2}$ of $S_{1}$ and $S_{2}$ as follows. Let $T(S)=T\left(S_{1}\right) \sqcup T\left(S_{2}\right)$ as sets. We define the map $\Delta(S): T(S) \rightarrow\{0,1\}$ to be $\left.\Delta(S)\right|_{T\left(S_{i}\right)}=\Delta\left(S_{i}\right)$ for $i=1,2$. Let $\Pi(S)$ be the map from $T(S)$ to itself satisfying that $\left.\Pi(S)\right|_{T\left(S_{i}\right)}=\Pi\left(S_{i}\right)$ for $i=1,2$. We define the order on $T(S)$ so that for elements $t$ and $t^{\prime}$ of $T(S)$,

(i) if $b(t) \leq b\left(t^{\prime}\right)$, then $t<t^{\prime}$, and

(ii) $t<t^{\prime}$ if and only if $\Pi(S)(t)<\Pi(S)\left(t^{\prime}\right)$ when $\Delta(S)(t)=\Delta(S)\left(t^{\prime}\right)$.

Notation 2.13. Let $N_{\xi}$ be the minimal $\mathrm{DM}_{1}$ of a Newton polygon $\xi=\sum_{i=1}^{z}\left(m_{i}, n_{i}\right)$. Let $S$ be the ABS associated to $N_{\xi}$. Then $S$ is described as $S=\bigoplus_{i=1}^{z} S_{i}$, where $S_{i}$ is the ABS associated to the $\mathrm{DM}_{1} N_{m_{i}, n_{i}}$. If an element $t$ of $T(S)$ belongs to $T\left(S_{r}\right)$, then we denote by $t^{r}$ or $\tau^{r}$ this element $t$ with $\tau=\Delta(S)(t)$. If we want to say that the element $t^{r}$ is the $i$-th element of $T\left(S_{r}\right)$, we write $t_{i}^{r}$ for the element $t^{r}$. Furthermore, we often write $\tau_{i}^{r}$ for the element $t_{i}^{r}$ of $T(S)$ with $\tau=\Delta(S)\left(t_{i}^{r}\right)$. Moreover, we describe the map $\Pi(S)$ by arrows:

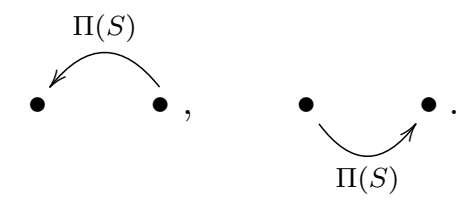

Example 2.14. Let us see an example of constructing a specialization. Let $\xi=(2,7)+$ $(3,5)$, and let $S$ be the ABS associated to $N_{\xi}$. Then $S$ is described as

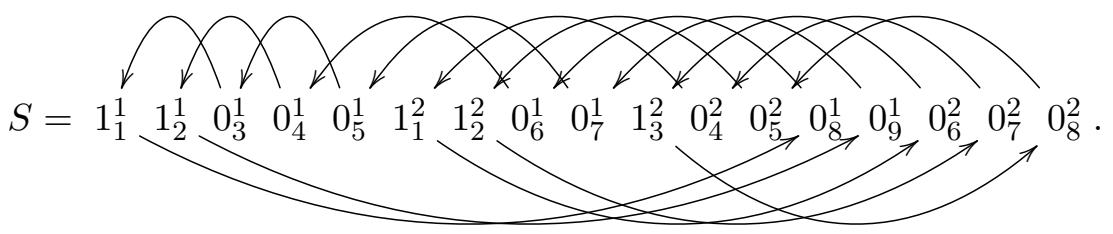

Let $S^{\prime}$ denote the specialization obtained by $\left(0_{4}^{1}, 1_{2}^{2}\right)$. Then $S^{\prime}$ is described as

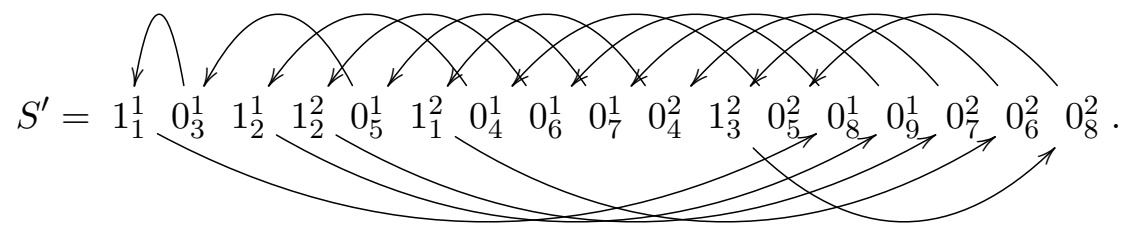


One can see that these $S$ and $S^{\prime}$ satisfy $\ell\left(S^{\prime}\right)=\ell(S)-1$, i.e., this $S^{\prime}$ is a generic specialization of $S$.

Example 2.15. Next, let us treat a Newton polygon consisting of three segments. Let $\xi=(2,7)+(1,2)+(3,5)$. Then the ABS $S$ corresponding to $N_{\xi}$ is

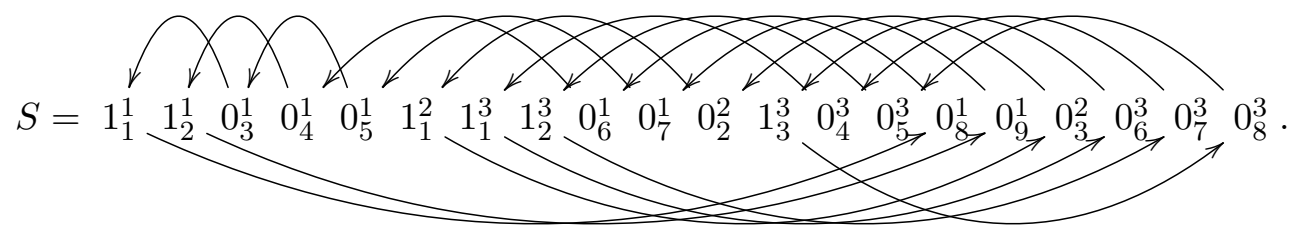

For this $S$, the specialization $S^{\prime}$ obtained by exchanging $0_{4}^{1}$ and $1_{2}^{3}$ is

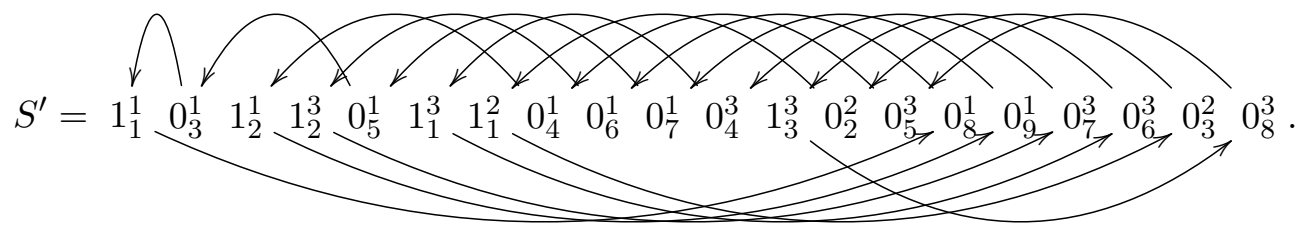

We see that this $S^{\prime}$ is not generic.

Example 2.16. Let $\xi=(3,4)+(3,2)$. Then the ABS $S$ corresponding to $N_{\xi}$ is described as

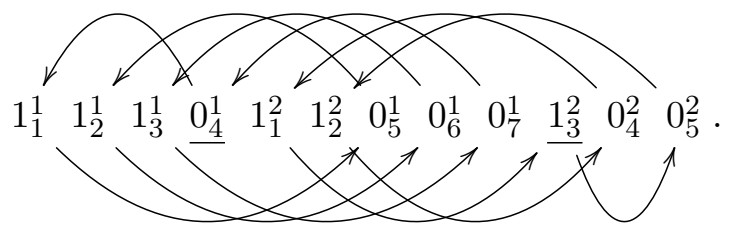

Let us consider the specialization obtained by $0_{4}^{1}$ and $1_{3}^{2}$. For elements $t$ of the small modification $T\left(S^{(0)}\right)$, binary expansions $b(t)$ are obtained by

$$
b(t)= \begin{cases}0.010101 \cdots & \text { if } \Delta(S)(t)=1 \\ 0.101010 \cdots & \text { otherwise. }\end{cases}
$$

Thus in this case, the specialization is not unique. However the $\mathrm{DM}_{1}$ corresponding the specialization is uniquely determined as $N_{\zeta}$ with $\zeta=6(1,1)$.

For certain Newton polygons $\xi$, the ABS associated to $N_{\xi}$ is described as follows:

Lemma 2.17. Let $N_{\xi}$ be the minimal $\mathrm{DM}_{1}$ of $\xi=\left(m_{1}, n_{1}\right)+\left(m_{2}, n_{2}\right)$ with $\lambda_{2}<1 / 2<\lambda_{1}$. For the above notation, the sequence $S$ associated to $N_{\xi}$ is obtained by the following:

$$
\underbrace{1_{1}^{1} \cdots 1_{m_{1}}^{1}}_{m_{1}} \underbrace{0_{m_{1}+1}^{1} \cdots 0_{n_{1}}^{1}}_{n_{1}-m_{1}} \underbrace{1_{1}^{2} \cdots 1_{n_{2}}^{2}}_{n_{2}} \underbrace{0_{n_{1}+1}^{1} \cdots 0_{h_{1}}^{1}}_{m_{1}} \underbrace{1_{n_{2}+1}^{2} \cdots 1_{m_{2}}^{2}}_{m_{2}-n_{2}} \underbrace{0_{m_{2}+1}^{2} \cdots 0_{h_{2}}^{2}}_{n_{2}} .
$$


Proof. See 2], Proposition 4.20.

In Definitions 2.18 and 2.19 below, we introduce sequences of sets, which are called $A$-sequences and $B$-sequences, to construct specializations $S^{\prime}$ of $S$ combinatorially. Using this method we can calculate lengths of specializations, and classify generic specializations. For instance, in Proposition 3.12 and Corollary 3.13, using this construction, we give a necessary condition for a specialization to be generic.

Definition 2.18. Let $S$ be the ABS of a minimal $\mathrm{DM}_{1}$. Let $S^{(0)}$ be the small modification by $\left(0_{i}^{r}, 1_{j}^{q}\right)$. Set $\delta=\Delta\left(S^{(0)}\right)$ and $\pi=\Pi\left(S^{(0)}\right)$. For non-negative integers $n$, we write $\alpha_{n}$ for $\pi^{n}\left(0_{i}^{r}\right)$. We define a subset $A_{0}$ of $T\left(S^{(0)}\right)$ to be

$$
A_{0}=\left\{t \in T\left(S^{(0)}\right) \mid t<\alpha_{0} \text { and } \alpha_{1}<\pi(t) \text { in } T\left(S^{(0)}\right), \text { with } \delta(t)=0\right\}
$$

endowed with the order induced from $T\left(S^{(0)}\right)$. Let $n$ be a natural number. We construct an $\operatorname{ABS} S^{(n)}$ and an ordered set $A_{n}$ by the ABS $S^{(n-1)}$ and the set $A_{n-1}$ as follows. Let $T\left(S^{(n)}\right)=T\left(S^{(n-1)}\right)$ as sets. We define the order on $T\left(S^{(n)}\right)$ so that for $t<t^{\prime}$ in $S^{(n-1)}$, we have $t>t^{\prime}$ if and only if $\alpha_{n}<t^{\prime} \leq \pi\left(t_{\max }\right)$ and $t=\alpha_{n}$ in $S^{(n-1)}$. Here $t_{\max }$ is the maximum element of $A_{n-1}$. We define the set $A_{n}$ by

$$
A_{n}=\left\{t \in T\left(S^{(n)}\right)-T\left(S_{q}\right) \mid t<\alpha_{n} \text { and } \alpha_{n+1}<\pi(t) \text { in } T\left(S^{(n)}\right) \text { with } \delta(t)=\delta\left(\alpha_{n}\right)\right\}
$$

endowed with the order induced from $S^{(n)}$. Thus we obtain the ABS $S^{(n)}=\left(T\left(S^{(n)}\right), \delta, \pi\right)$ and the set $A_{n}$. We call $\left\{A_{n}\right\}$ the $A$-sequence associated to $S, 0_{i}^{r}$ and $1_{j}^{q}$.

Proposition 3.8 implies that if the specialization obtained by a small modification is generic, then there exists a non-negative integer $a$ such that $A_{a}=\emptyset$. Now we suppose that there exists such an integer $a$. Then we can define the following ABS's and sets.

Definition 2.19. For the ABS $S$ corresponding to a minimal $\mathrm{DM}_{1}$, let $S^{(0)}$ be the small modification by $\left(0_{i}^{r}, 1_{j}^{q}\right)$. We write $\delta$ for $\Delta\left(S^{(0)}\right)$ and $\pi$ for $\Pi\left(S^{(0)}\right)$. Put $\beta_{n}=\pi^{n}\left(1_{j}^{q}\right)$ for non-negative integers $n$. Assume that there exists the minimum non-negative integer $a$ such that $A_{a}=\emptyset$, and we define a set $B_{0}$ by

$$
B_{0}=\left\{t \in T\left(S^{(a)}\right) \mid \beta_{0}<t \text { and } \pi(t)<\beta_{1} \text { in } T\left(S^{(a)}\right) \text { with } \delta(t)=1\right\}
$$

endowed with the order induced from $T\left(S^{(a)}\right)$. For the ABS $S^{(a+n-1)}$ and the set $B_{n-1}$, we define an $\operatorname{ABS} S^{(a+n)}$ as follows. Let $T\left(S^{(a+n)}\right)=T\left(S^{(a+n-1)}\right)$ as sets. Let $\Delta\left(S^{(a+n)}\right)=$ $\Delta\left(S^{(a+n-1)}\right)$ and $\Pi\left(S^{(a+n)}\right)=\Pi\left(S^{(a+n-1)}\right)$. The ordering of $T\left(S^{(a+n)}\right)$ is given so that for $t<t^{\prime}$ in $S^{(a+n-1)}$, we have $t>t^{\prime}$ if and only if $\pi\left(t_{\min }\right) \leq t<\beta_{n}$ and $t^{\prime}=\beta_{n}$, where $t_{\min }$ is the minimum element of $B_{n-1}$. We define an ordered set $B_{n}$ as

$$
B_{n}=\left\{t \in T\left(S^{(a+n)}\right) \mid \beta_{n}<t \text { and } \pi(t)<\beta_{n+1} \text { in } T\left(S^{(a+n)}\right) \text { with } \delta(t)=\delta\left(\beta_{n}\right)\right\}
$$


with the ordering obtained from the order on $S^{(a+n)}$. Thus we obtain the ABS $S^{(a+n)}$ and the set $B_{n}$. We call $\left\{B_{n}\right\}$ the $B$-sequence associated to $S, 0_{i}^{r}$ and $1_{j}^{q}$.

For a small modification by $\left(0_{i}^{r}, 1_{j}^{q}\right)$, if there exists non-negative integers $a$ and $b$ such that $A_{a}=\emptyset$ and $B_{b}=\emptyset$, then we call the ABS $S^{(a+b)}$ the full modification by $\left(0_{i}^{r}, 1_{j}^{q}\right)$. In Proposition 3.10, we will see that if the specialization is generic, then for the above sets $B_{n}$, there exists a non-negative integer $b$ such that $B_{b}=\emptyset$, i.e., every generic specialization is obtained by the ABS $S^{(a+b)}$ for some integers $a$ and $b$. The small modification in Example 2.16 is an example where the number $b$ does not exist. The specialization of the ABS $S$ of Example 2.16 is obtained by $S^{(5)}$.

Example 2.20. Let $\xi=(2,7)+(3,5)$. Let $S$ be the ABS of $N_{\xi}$. The small modification $S^{(0)}$ by $\left(0_{4}^{1}, 1_{2}^{2}\right)$ is described as

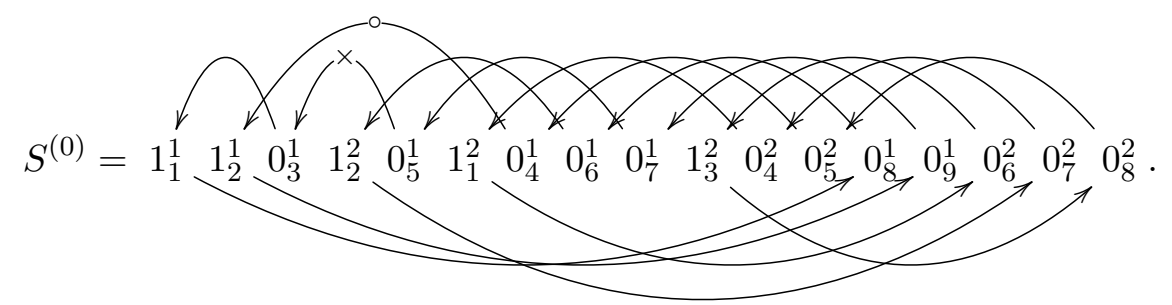

For this $S^{(0)}$, we have sets $A_{0}=\left\{0_{5}^{1}\right\}$ and $A_{1}=\emptyset$. The ABS $S^{(1)}$ is obtained by

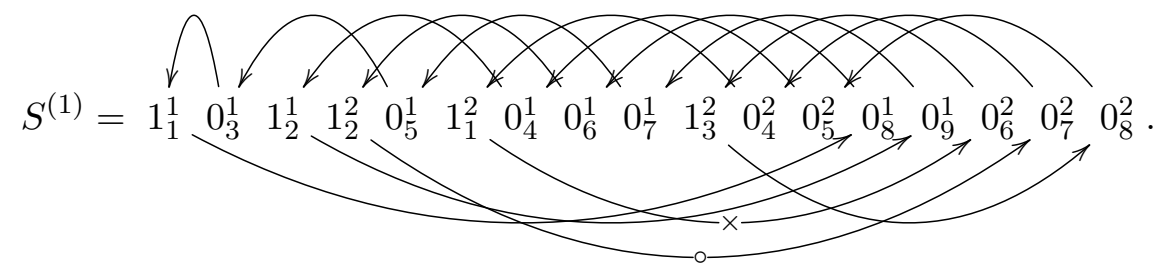

By the above $B_{0}=\left\{1_{1}^{2}\right\}$. We have $B_{1}=\left\{0_{7}^{2}\right\}$ with the ABS

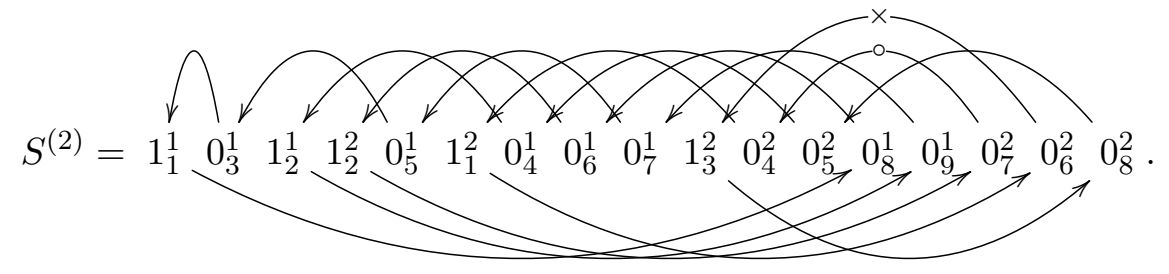

Clearly $B_{2}=\emptyset$. Hence we see $a=1$ and $b=1$. One can check that the full modification $S^{(3)}$ is equal to $S^{\prime}$ of Example 2.14 .

Example 2.21. Let $\xi=(3,10)+(5,2)$. The ABS corresponding to the $\mathrm{DM}_{1} N_{\xi}$ is described as 


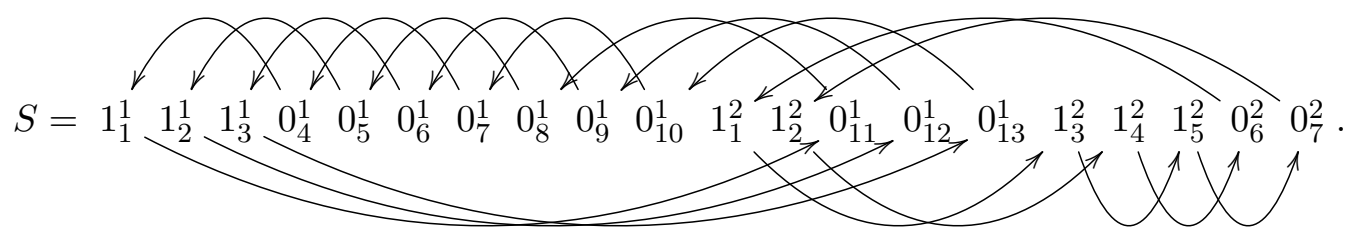

Let us treat the small modification $S^{(0)}$ obtained by $\left(00_{8}^{1}, 1_{2}^{2}\right)$. Then $S^{(0)}$ is

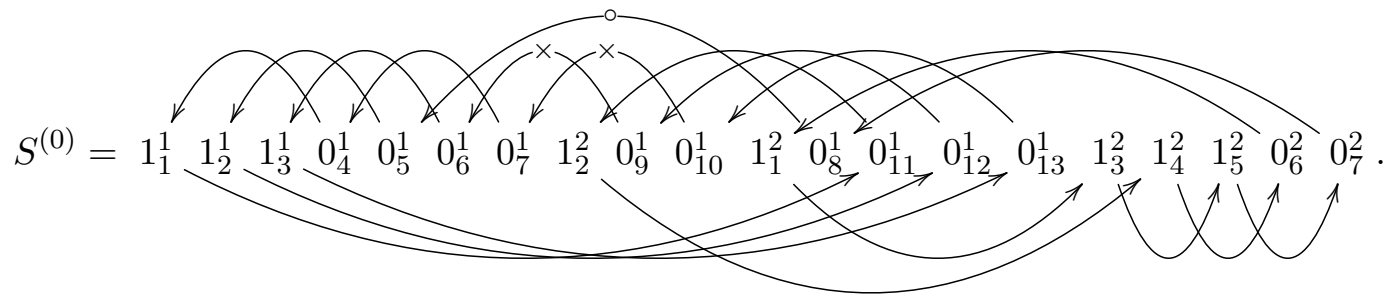

We have then $A_{0}=\left\{00_{9}^{1}, 0_{10}^{1}\right\}$. The $\operatorname{ABS} S^{(1)}$ is

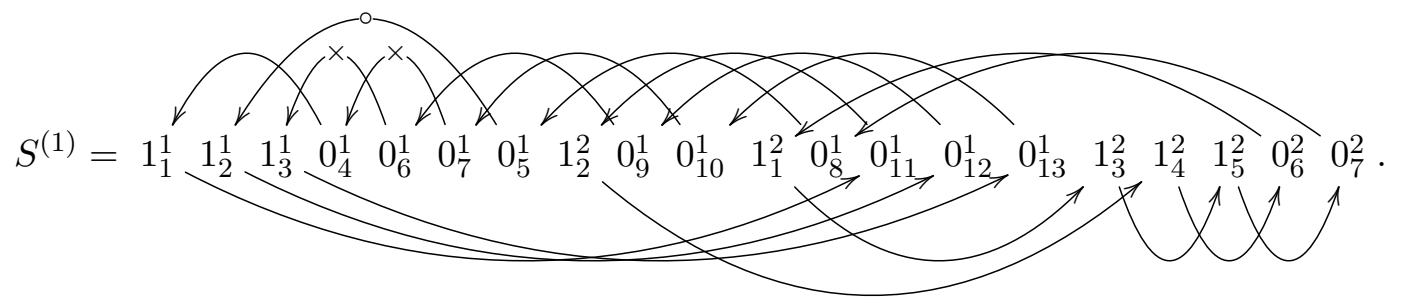

We have $A_{1}=\left\{0_{6}^{1}, 0_{7}^{1}\right\}$. The ABS $S^{(2)}$ is

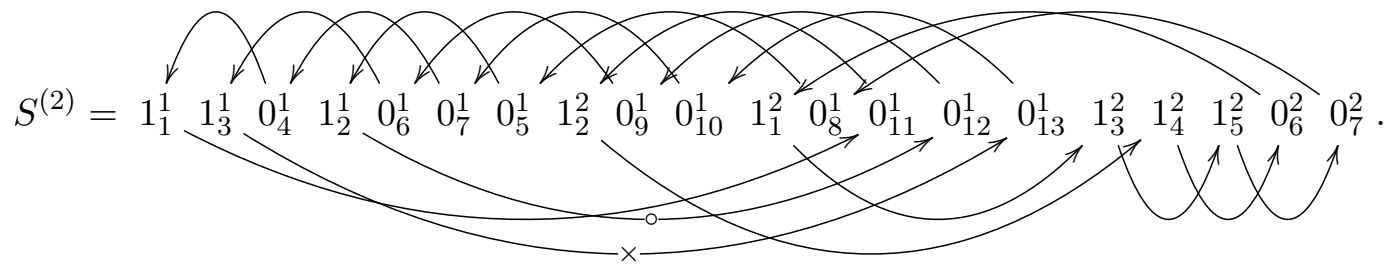

The set $A_{2}$ is $\left\{1_{3}^{1}\right\}$. Similarly, we have $A_{3}=\left\{0_{13}^{1}\right\}, A_{4}=\left\{0_{10}^{1}\right\}, A_{5}=\left\{0_{7}^{1}\right\}$ and $A_{6}=\emptyset$. The $\operatorname{ABS} S^{(6)}$ is

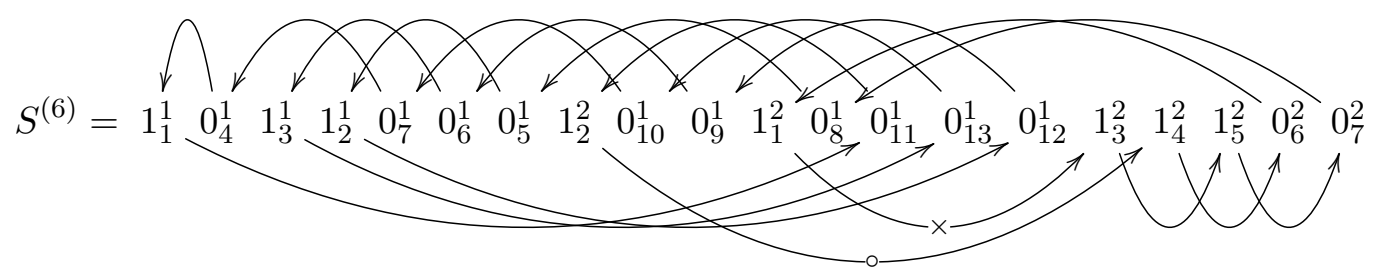

with $B_{0}=\left\{1_{1}^{2}\right\}$. By definition, we have $B_{1}=\left\{1_{3}^{2}\right\}$, and the set $B_{2}$ is an empty set. Thus we obtain $a=6$ and $b=2$, and the full modification $S^{(8)}$ is described as

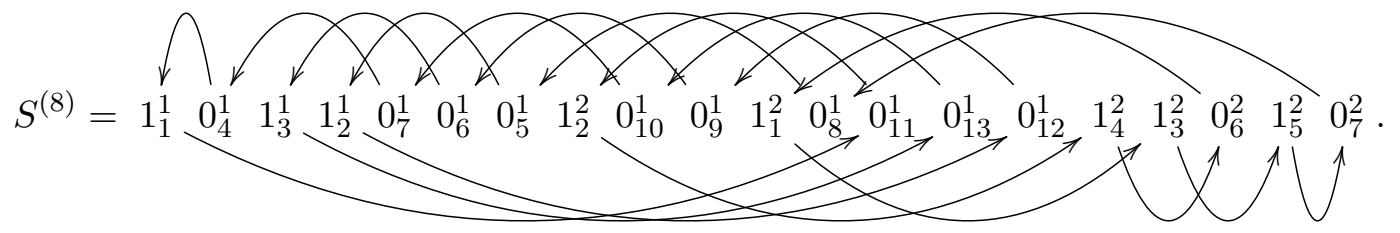


One can see that this full modification satisfies that $\ell\left(S^{(8)}\right)=\ell(S)-1$.

See also Example 3.14 for an example of constructing a full modification.

\section{The case of arbitrary Newton polygons}

Let $\xi$ be a Newton polygon. In this section, we will prove Theorem 1.5 , and reduce Problem 1.4 to the case that $\xi$ consists of two slopes. Let $S$ be the ABS corresponding to the minimal $\mathrm{DM}_{1} N_{\xi}$, and let $S^{\prime}$ be the specialization of $S$ obtained by $\left(0_{i}^{r}, 1_{j}^{q}\right)$ with $r<q$. The key statement is that $S^{\prime}$ is generic only if the $r$-th segment is adjacent to the $q$-th segment, i.e., $q=r+1$, see Corollary 3.13. Moreover, to show this, we give some necessary conditions for a specialization to be generic in Propositions 3.8 and 3.10 .

\subsection{Euclidean algorithm for Newton polygons}

We denote by NP the set of Newton polygons satisfying that the segments of a Newton polygon are not all the same. Let $\mathrm{NP}^{\mathrm{sep}}$ be the subset of NP consisting of Newton polygons $\sum_{i=1}^{z}\left(m_{i}, n_{i}\right)$ with $n_{z} /\left(m_{z}+n_{z}\right)<1 / 2<n_{1} /\left(m_{1}+n_{1}\right)$. In this section, we introduce a map from NP to NP ${ }^{\text {sep }}$. In Proposition 3.5, using this map, we show a property of ABS's corresponding to minimal $\mathrm{DM}_{1}$ 's. Moreover, thanks to this map, to solve Problem 1.4, we may assume that the Newton polygon belongs to NPsep and consists of two segments, see Section 4. To introduce the map, first, we define two operations of Newton polygons. Let $\xi=\sum_{i=1}^{z}\left(m_{i}, n_{i}\right)$ be a Newton polygon. We define the Newton polygon $\xi^{\mathrm{D}}$ by

$$
\xi^{\mathrm{D}}=\sum_{i=1}^{z}\left(n_{z-i+1}, m_{z-i+1}\right) .
$$

We call this $\xi^{\mathrm{D}}$ the dual of $\xi$. Moreover, if $\xi$ satisfies $m_{i} \leq n_{i}$ for all $i$, we define the Newton polygon $\xi^{\mathrm{C}}$ by

$$
\xi^{\mathrm{C}}=\sum_{i=1}^{z}\left(m_{i}, n_{i}-m_{i}\right),
$$

and we call this $\xi^{\mathrm{C}}$ the curtailment of $\xi$.

For the Newton polygon $\xi$, we define the height of $\xi$ by $h t(\xi)=m_{1}+n_{1}+m_{2}+n_{2}+$ $\cdots+m_{z}+n_{z}$. Let $\xi$ belong to NP. Let us define a map $\Phi: \mathrm{NP} \rightarrow \mathrm{NP}^{\text {sep }}$ by induction on the height of $\xi$. First we assume $z=2$. If $\xi$ belongs to $\mathrm{NP}^{\mathrm{sep}}$, then define $\Phi(\xi)=\xi$. Note that the segments $\left(m_{i}, n_{i}\right)$ are different from each other by the definition of NP. So for at least one segment $\left(m_{i}, n_{i}\right)$, we have $m_{i}<n_{i}$ or $n_{i}<m_{i}$. If $m_{i} \leq n_{i}$ for $i=1,2$, then we define the image $\Phi(\xi)$ of $\xi$ to be the image of $\Phi$ of $\xi^{\mathrm{C}}$. On the other hand, if $m_{i} \geq n_{i}$ for $i=1,2$, then we define the image $\Phi(\xi)$ to be the image of $\Phi$ of $\xi^{\mathrm{DC}}$. Let us see that this operation ends in a finite number of times. It is obvious that $\operatorname{ht}\left(\xi^{\mathrm{C}}\right)<\operatorname{ht}(\xi)$ 
and $\operatorname{ht}\left(\xi^{\mathrm{D}}\right)=\operatorname{ht}(\xi)$. Since $\operatorname{gcd}\left(m_{i}, n_{i}\right)=1$ for all $i$, we see that the height of $\xi$ is greater than one. If $\operatorname{ht}(\xi)=2$, then, since $\xi$ has at least two types of segments, we see that $\xi$ is uniquely determined by $\xi=(0,1)+(1,0)$.

Finally, we treat the case $z>2$. For the Newton polygon $\eta=\left(m_{1}, n_{1}\right)+\left(m_{z}, n_{z}\right)$, let $\mathrm{W}$ be the word of $\mathrm{C}$ and $\mathrm{D}$ such that $\Phi(\eta)=\eta^{\mathrm{W}}$. We define the image $\Phi(\xi)$ of $\xi$ by $\xi^{\mathrm{W}}$.

Remark 3.1. By the above construction, the Newton polygon $\Phi(\xi)$ is described as $\Phi(\xi)=$ $\xi^{\mathrm{Q}_{1} \mathrm{Q}_{2} \cdots \mathrm{Q}_{m}}$, where $\mathrm{Q}_{i}$ is either the operation $\mathrm{C}$ or the operation $\mathrm{D}$ for every $i$. Thus by the duality, if Theorem 1.6 is true, then for all Newton polygons $\xi$ consisting of two segments, we obtain a bijection from $B(\xi)$ to $B(\Phi(\xi))$.

Example 3.2. Let $\xi=(5,13)+(7,16)$. Then $\Phi(\xi)$ is obtained by $\Phi(\xi)=\xi^{\mathrm{CCDC}}=$ $(2,5)+(3,2)$.

Let $S$ (resp. $R$ ) be the ABS of $N_{\xi}$ (resp. $N_{\xi^{\mathrm{C}}}$ ). Next, we describe a relation between $S$ and $R$. In the following lemma, we show that the set $T(R)$ can be regarded as a subset of $T(S)$ as ordered sets. This relation is used for the proof of Proposition 3.5. Lemma 3.11 and Theorem 1.6 .

Lemma 3.3. Let $\xi=\left(m_{1}, n_{1}\right)+\left(m_{2}, n_{2}\right)$ be a Newton polygon consisting of two segments with $m_{i} \leq n_{i}$ for $i=1,2$. Let $S$ and $R$ be the $A B S$ 's corresponding to $N_{\xi}$ and $N_{\xi^{\mathrm{C}}}$ respectively. Put $T(S)=\left\{t_{1}, \ldots, t_{h}\right\}$ and $T(R)=\left\{t_{1}^{\prime}, \ldots, t_{h^{\prime}}^{\prime}\right\}$ with $h=m_{1}+n_{1}+m_{2}+n_{2}$ and $h^{\prime}=n_{1}+n_{2}$. We regard $T(R)$ as a subset of $T(S)$ as ordered sets by the map sending $t_{i}^{\prime}$ to $t_{i}$. Then we have

$$
\{t \in T(R) \mid \Delta(R)(t)=1\}=\{t \in T(S) \mid \Delta(S)(t)=1\}
$$

as ordered sets, and

$$
T(S)-T(R)=\{\Pi(S)(t) \mid t \in T(S) \text { with } \Delta(S)(t)=1\}
$$

Let $t$ be an element of $T(R)$. We also regard $t$ as an element of $T(S)$. Then

$$
\Pi(R)(t)= \begin{cases}\Pi(S)(t) & \text { if } \Delta(R)(t)=0 \\ \Pi(S)^{2}(t) & \text { otherwise }\end{cases}
$$

holds.

Proof. Recall that for the $i$-th element $t_{i}^{r}$ of $T\left(S_{r}\right)$, with $S_{r}$ the ABS corresponding to the $\mathrm{DM}_{1} N_{m_{r}, n_{r}}$, we have $\Pi\left(S_{r}\right)\left(t_{i}^{r}\right)=t_{i+n_{r}}^{r}$ if $\Delta\left(S_{r}\right)\left(t_{i}\right)=1$ and $\Pi\left(S_{r}\right)\left(t_{i}^{r}\right)=t_{i-m_{r}}^{r}$ otherwise. Since $\xi^{\mathrm{C}}=\left(m_{1}, n_{1}-m_{1}\right)+\left(m_{2}, n_{2}-m_{2}\right)$, it is clear that $T(R)$ is a subset of $T(S)$ as sets, and we have the standard one-to-one correspondence between $\{t \in T(R) \mid \Delta(R)(t)=1\}$ 
and $\{t \in T(S) \mid \Delta(S)(t)=1\}$ as sets. Let $t$ and $s$ be elements of $\{t \in T(R) \mid \Delta(R)(t)=1\}$. Let $t^{\prime}$ and $s^{\prime}$ be the elements of $\{t \in T(S) \mid \Delta(S)(t)=1\}$ corresponding to $t$ and $s$ respectively by the standard one-to-one correspondence. Considering binary expansions, one can see that $b(t)<b(s)$ if and only if $b\left(t^{\prime}\right)<b\left(s^{\prime}\right)$. Indeed, the value replacing 1 with 01 for $b(t)$ is equal to $b\left(t^{\prime}\right)$. Put $b(t)=0 . b_{1} b_{2} \ldots$ and $b(s)=0 . b_{1}^{\prime} b_{2}^{\prime} \ldots$ We have $b(t)<b(s)$ if and only if there exists a non-negative integer $v$ such that $b_{l}=b_{l}^{\prime}$ for $l<v$ and $b_{v}<b_{v}^{\prime}$. Hence clearly we have $b\left(t^{\prime}\right)<b\left(s^{\prime}\right)$. Thus we obtain the equality (3.1) as ordered sets, and this implies that $T(R)$ is a subset of $T(S)$ as ordered sets. We immediately obtain the equalities 3.2 and 3.3 .

Note that all elements t of $T(S)-T(R)$ satisfy $\Delta(S)(t)=0$. Example 3.4 below is an example of Lemma 3.3 .

Example 3.4. Let $\xi=(2,7)+(3,5)$. Let $S$ and $R$ be the ABS's corresponding to $N_{\xi}$ and $N_{\xi^{\mathrm{C}}}$ respectively. We have then

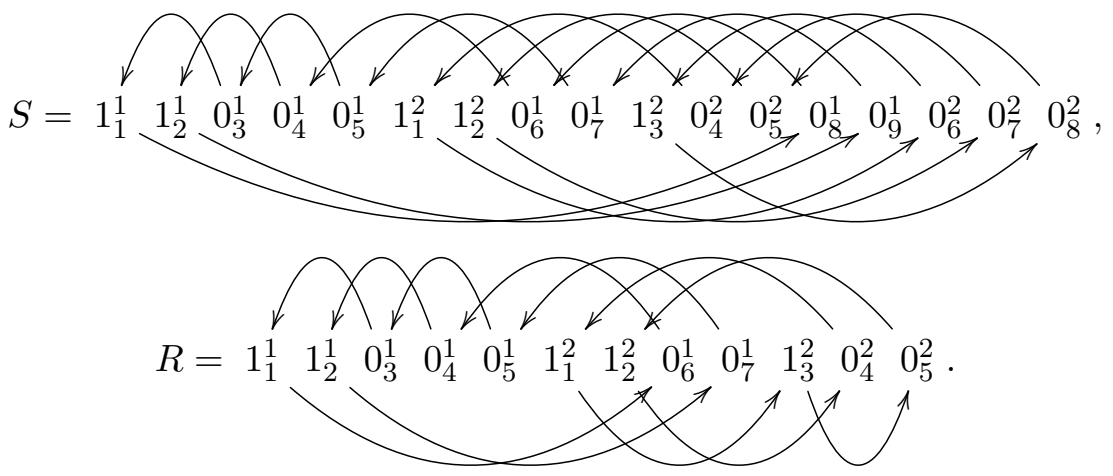

One can easily check that the equations (3.1), 3.2) and (3.3) of Lemma 3.3 hold.

Thanks to the map $\Phi: \mathrm{NP} \rightarrow \mathrm{NP}^{\text {sep}}$, we can partially describe the construction of the ABS corresponding to $N_{\xi}$ for a Newton polygon $\xi$. We state it as follows:

Proposition 3.5. Let $S$ be the $A B S$ of a minimal $\mathrm{DM}_{1} N_{\xi}$ with $\xi=\sum_{i=1}^{z}\left(m_{i}, n_{i}\right)$. For natural numbers $r$ and $q$ with $r<q$, we have
(i) $1_{1}^{r}<1_{1}^{q}$,
(ii) $0_{m_{r}+n_{r}}^{r}<0_{m_{q}+n_{q}}^{q}$,
(iii) $0_{m_{r}+1}^{r}<0_{m_{q}+1}^{q}$

in the ordered set $T(S)$.

Proof. To show the proposition, it suffices to deal with the case $z=2$. Note that (iii) follows from (i) since $0_{m_{r}+1}^{r}$ and $0_{m_{q}+1}^{q}$ are the inverse images of $1_{1}^{r}$ and $1_{1}^{q}$ by $\Pi(S)$ respectively. For a Newton polygon $\xi$, let $\mathrm{P}(\xi)$ denote the assertion: The ABS associated to the minimal $\mathrm{DM}_{1} N_{\xi}$ satisfies (i) and (ii). By Lemma 2.17, if $\xi$ satisfies that $\lambda_{2}<$ $1 / 2<\lambda_{1}$, then $\mathrm{P}(\xi)$ holds. To show that $\mathrm{P}(\xi)$ is true for all Newton polygons $\xi$, we claim 
(A) If $\mathrm{P}\left(\xi^{\mathrm{D}}\right)$ holds, then $\mathrm{P}(\xi)$ also holds;

(B) If $m_{i} \leq n_{i}$ for all $i$ and $\mathrm{P}\left(\xi^{\mathrm{C}}\right)$ holds, then $\mathrm{P}(\xi)$ also holds.

The claim (A) is obvious by the duality. Moreover, by Lemma 3.3, we see that (B) holds. In fact, let $R$ be the ABS corresponding to $N_{\xi^{\mathrm{C}}}$. Clearly $1_{1}^{1}<1_{1}^{2}$ holds in $T(S)$ by Lemma 3.3. Moreover, in $T(S)$, the inverse images $0_{m_{1}+n_{1}}^{1}$ and $0_{m_{2}+n_{2}}^{2}$ of $0_{n_{1}}^{1}$ and $0_{n_{2}}^{2}$ respectively by $\Pi(S)$ satisfy that $0_{m_{1}+n_{1}}^{1}<0_{m_{2}+n_{2}}^{2}$ since in $T(R)$ we have $0_{n_{1}}^{1}<0_{n_{2}}^{2}$. For a Newton polygon $\xi$, we have the Newton polygon $\Phi(\xi)$ satisfying $\lambda_{2}<1 / 2<\lambda_{1}$ using the map $\Phi: \mathrm{NP} \rightarrow \mathrm{NP}^{\mathrm{sep}}$, whence the assertion of the proposition follows from (A) and (B).

\subsection{Combinatorial construction of generic specializations}

In this section, using $A$-sequences and $B$-sequences defined in Definitions 2.18 and 2.19 respectively, we construct a full modification, which is a specialization constructed combinatorially, for a given small modification.

We use the notation of Notation 2.13. Furthermore, we fix the following notation. Let $S$ be the ABS associated to $N_{\xi}$. Let $S^{(0)}$ be the small modification by $\left(0_{i}^{r}, 1_{j}^{q}\right)$ with $r<q$. Then we obtain arrowed binary sequences $S^{(1)}, S^{(2)}, \ldots$ and the $A$-sequence $\left\{A_{n}\right\}$ by Definition 2.18. Put $\delta=\Delta\left(S^{(0)}\right)$ and $\pi=\Pi\left(S^{(0)}\right)$. We set $\alpha_{n}=\pi^{n}\left(0_{i}^{r}\right)$ and $\beta_{n}=\pi^{n}\left(1_{j}^{q}\right)$ for non-negative integers $n$.

Proposition 3.6. Let $a^{\prime}$ be the natural number satisfying that $\alpha_{a^{\prime}}=\beta_{0}$. Let $n$ be a natural number with $n<a^{\prime}$. The set $A_{n}$ is equal to

$$
\left\{\pi(t) \mid t \in A_{n-1}, \pi(t) \notin T\left(S_{q}\right) \text { and } \delta(\pi(t))=\delta\left(\alpha_{n}\right)\right\} \text {. }
$$

Proof. Note that, for elements $t$ and $t^{\prime}$ of $T\left(S^{(n)}\right)$, with $n<a^{\prime}$, we have $t<t^{\prime}$ and $\pi\left(t^{\prime}\right)<\pi(t)$ with $\delta(t)=\delta\left(t^{\prime}\right)$ if and only if $t \in A_{n}$ and $t^{\prime}=\alpha_{n}$, or $t=\beta_{0}$ and $t^{\prime} \in B_{0}^{\prime}$, where $B_{0}^{\prime}=\left\{t \in T\left(S^{(0)}\right) \mid \beta_{0}<t\right.$ and $\pi(t)<\beta_{1}$ in $T\left(S^{(0)}\right)$ with $\left.\delta(t)=1\right\}$. First, take an element $\pi(t)$ of the set $(3.4)$. Let us show that this $\pi(t)$ belongs to $A_{n}$. We describe the part of $S^{(n-1)}$ as

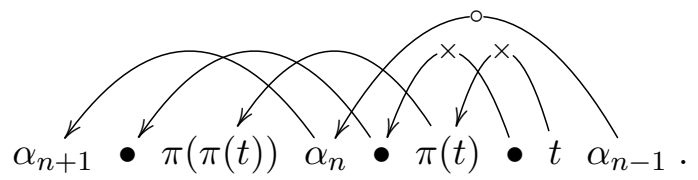

Since $t$ belongs to $A_{n-1}$, we see $\alpha_{n}<\pi(t)$ in $T\left(S^{(n-1)}\right)$. As $\delta(\pi(t))=\delta\left(\alpha_{n}\right)$, this induces $\alpha_{n+1}<\pi(\pi(t))$ in $T\left(S^{(n-1)}\right)$. To construct $S^{(n)}$, we move the element $\alpha_{n}$ to the right side of the maximum element of $\pi\left(A_{n-1}\right)$. By construction, the part of $S^{(n)}$ can be described as 


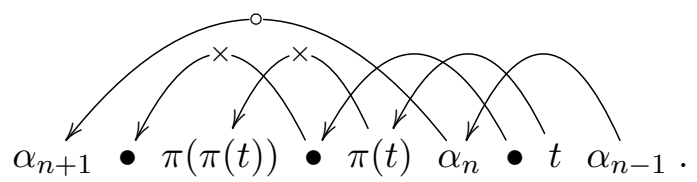

We have then $\pi(t)<\alpha_{n}$ and $\alpha_{n+1}<\pi(\pi(t))$ in $S^{(n)}$. Hence we see that $\pi(t)$ belongs to $A_{n}$.

Conversely, take an element $t^{\prime}$ of $A_{n}$. There exists the element $t$ of $T\left(S^{(n)}\right)$ such that $t^{\prime}=\pi(t)$. It suffices to show that $t$ belongs to $A_{n-1}$. This $t$ satisfies that $\pi(t)<\alpha_{n}$ and $\alpha_{n+1}<\pi(\pi(t))$ in $T\left(S^{(n)}\right)$ by the definition of $A_{n}$. Since $\delta(\pi(t))=\delta\left(\alpha_{n}\right)$, we have $\alpha_{n+1}<\pi(\pi(t))$ and $\alpha_{n}<\pi(t)$ in $T\left(S^{(n-1)}\right)$. On the other hand, to lead a contradiction, suppose $\alpha_{n-1}<t$ in $T\left(S^{(n-1)}\right)$. Then for the maximum element $t_{\max }$ of $A_{n-1}$, since $\alpha_{n}<\pi\left(t_{\max }\right)<\pi(t)$ in $T\left(S^{(n-1)}\right)$, we have $\alpha_{n}<\pi(t)$ in $T\left(S^{(n)}\right)$ by the construction of $A_{n}$. This is a contradiction. Thus we have shown that $t<\alpha_{n-1}$ in $T\left(S^{(n-1)}\right)$, and $t$ belongs to $A_{n-1}$. Hence we see that $t^{\prime}$ belongs to the set (3.4).

Proposition 3.7. Let $n$ be a non-negative integer. $A_{n}$ does not contain elements $\alpha_{m}$ for $m \leq n$.

Proof. Note that for every non-negative integer $n$, by the definition of $A_{n}$, the set $A_{n}$ does not contain the inverse image $\alpha_{-1}$ of $\alpha_{0}$ by $\Pi\left(S^{(0)}\right)$ as $\alpha_{-1}$ is an element of $T\left(S_{q}\right)$. Here $S_{q}$ is the ABS corresponding to the $\mathrm{DM}_{1} N_{m_{q}, n_{q}}$. Let us show the assertion by induction on $n$. The case $n=0$ is obvious. For a natural number $n$, to lead a contradiction, suppose that $A_{n}$ contains $\alpha_{m}$ for a non-negative integer $m$ with $m \leq n$. By Proposition 3.6, then $A_{n-1}$ contains $\alpha_{m-1}$. This contradicts the hypothesis of induction.

Proposition 3.8. If there exists no non-negative integer a such that $A_{a}=\emptyset$, then the specialization $S^{\prime}$ is not generic.

Proof. First, let us construct the specialization combinatorially in this case. Let $a^{\prime}$ be the minimum number satisfying $\alpha_{a^{\prime}}=\beta_{0}$. Let $B_{0}^{\prime}$ be the subset of $T\left(S^{(0)}\right)$

$$
B_{0}^{\prime}=\left\{t \in T\left(S^{(0)}\right) \mid \beta_{0}<t \text { and } \pi(t)<\beta_{1} \text { in } T\left(S^{(0)}\right) \text { with } \delta(t)=1\right\} .
$$

A part of the ABS $S^{\left(a^{\prime}-1\right)}$ can be described as

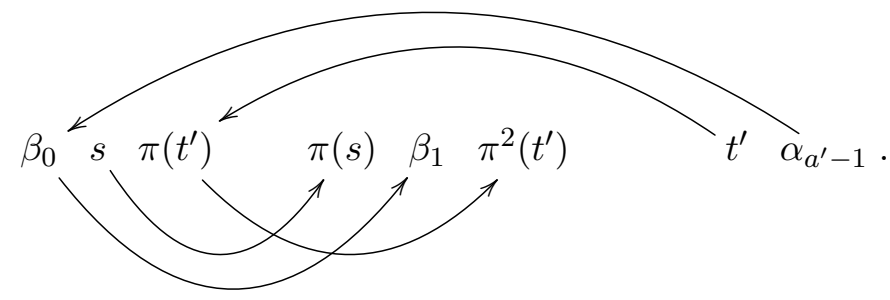

Suppose that $\pi\left(A_{a^{\prime}-1}\right)$ is contained in $B_{0}^{\prime}$. Then, since $t^{\prime \prime}<\beta_{0}$ and $\pi\left(t^{\prime \prime}\right)<\beta_{1}$ in $T\left(S^{\left(a^{\prime}\right)}\right)$ for all elements $t^{\prime \prime}$ of $\pi\left(A_{a^{\prime}-1}\right)$, there is no element $s \in T\left(S^{\left(a^{\prime}\right)}\right)$ such that $s<\alpha_{a^{\prime}}$ 
and $\alpha_{a^{\prime}+1}<\pi(s)$, i.e., $A_{a^{\prime}}$ is empty. This is a contradiction. Thus there exists an element $t^{\prime}$ of $A_{a^{\prime}-1}$ such that $\beta_{0}<\pi\left(t^{\prime}\right)$ and $\beta_{1}<\pi\left(\pi\left(t^{\prime}\right)\right)$ in $T\left(S^{\left(a^{\prime}-1\right)}\right)$ with $\delta\left(\beta_{0}\right)=\delta\left(\pi\left(t^{\prime}\right)\right)$. Then for all elements $s$ of $B_{0}^{\prime}$, we have $s<\pi\left(t^{\prime}\right)$. In fact, if $\pi\left(t^{\prime}\right)<s$, then $\pi\left(t^{\prime}\right)<s$ and $\pi(s)<\pi\left(\pi\left(t^{\prime}\right)\right)$ holds with $\delta(s)=\delta\left(\pi\left(t^{\prime}\right)\right)$, and this is a contradiction. Thus there exists no element $u$ of $T\left(S^{\left(a^{\prime}\right)}\right)$ such that $\beta_{0}<u$ and $\pi(u)<\beta_{1}$. This implies that elements $t$ and $t^{\prime}$ of $T\left(S^{\left(a^{\prime}\right)}\right)$ with $t<t^{\prime}$ satisfy $\pi\left(t^{\prime}\right)<\pi(t)$ if and only if $t^{\prime}=\alpha_{a^{\prime}}$ and $t \in A_{a^{\prime}}$. By hypothesis, there exists a non-negative integer $m$ such that $\left|A_{m}\right|=\left|A_{m+1}\right|=\cdots$. By the definition of the sets $A_{n}$, for $n \geq m$, all elements $t$ of $A_{n}$ have the same value $\delta(t)$. For this number $m$, the elements of $T\left(S^{(m)}\right)$ are ordered by binary expansions by $\pi$. Let $S^{\prime}$ be the admissible ABS associated to $\left(\delta\left(t_{1}\right), \ldots, \delta\left(t_{h}\right)\right)$ with $T\left(S^{(m)}\right)=\left\{t_{1}<\cdots<t_{h}\right\}$. Then $S^{\prime}$ is a specialization of $S$. Note that $\ell\left(S^{\prime}\right)=\ell\left(S^{(m)}\right)$.

Here, let us compare lengths of $S$ and $S^{\prime}$. One can see that $\ell(S)-\ell\left(S^{(0)}\right)=\left|A_{0}\right|+$ $\left|B_{0}^{\prime}\right|+1$. Since $\ell\left(S^{(m)}\right)-\ell\left(S^{(0)}\right) \leq\left|A_{0}\right|-\left|A_{m}\right|$, we see $\ell\left(S^{\prime}\right)<\ell(S)-1$.

By Proposition 3.8, we may assume that there exists a non-negative integer a such that $A_{a}$ is an empty set to classify generic specializations $S^{\prime}$ of $S$.

In Proposition 3.10 below, we will show that to classify generic specializations, it suffices to consider the case that there exists a non-negative integer $b$ such that $B_{b}=\emptyset$. Note that, to show Proposition 3.10, we may assume that there exists a non-negative integer $a$ such that $A_{a}=\emptyset$. Let us see a property of the $B$-sequence, which is used for the proof of Proposition 3.10 .

Proposition 3.9. Let $n$ be a natural number. The set $B_{n}$ is equal to

$$
\left\{\pi(t) \mid t \in B_{n-1} \text { and } \delta(\pi(t))=\delta\left(\beta_{n}\right)\right\}
$$

Proof. Note that the $B$-sequence is the "dual" object of the $A$-sequence. A proof is given by the same way as Proposition 3.6 .

Proposition 3.10. If there exists no non-negative integer $b$ such that $B_{b}=\emptyset$, then the specialization is not generic.

Proof. In this hypothesis, there exists a non-negative integer $m$ such that $\left|B_{m}\right|=\left|B_{m+1}\right|=$ $\cdots$. By the definition of the sets $B_{n}$, for $n \geq m$, all elements $t$ of $B_{n}$ have the same value $\delta(t)$. Then the elements of $T\left(S^{(a+m)}\right)$ are ordered by these binary expansions by $\pi$. Let $S^{\prime}$ be the admissible ABS associated to $\left(\delta\left(t_{1}\right), \ldots, \delta\left(t_{h}\right)\right)$ with $T\left(S^{(a+m)}\right)=\left\{t_{1}<\cdots<t_{h}\right\}$. Then $S^{\prime}$ is a specialization of $S$. Note that $\ell\left(S^{\prime}\right)=\ell\left(S^{(a+m)}\right)$.

Let us compare lengths of $S$ and $S^{\prime}$. It is clear that $\ell(S)-\ell\left(S^{(0)}\right)=\left|A_{0}\right|+\left|B_{0}^{\prime}\right|+1$, where the set $B_{0}^{\prime}$ is same as in Proposition 3.8. If the non-negative integer $a$ satisfies $a \geq a^{\prime}$ with the minimum number $a^{\prime}$ satisfying $\alpha_{a^{\prime}}=\beta_{0}$, then $\left|B_{0}\right|<\left|B_{0}^{\prime}\right|$ by the proof of 
Proposition 3.8. Since $\ell\left(S^{(a)}\right)-\ell\left(S^{(0)}\right) \leq\left|A_{0}\right|$ and $\ell\left(S^{(a+m)}\right)-\ell\left(S^{(a)}\right) \leq\left|B_{0}\right|-\left|B_{m}\right|$, we see that the specialization $S^{\prime}$ is not generic in this case. Let us see the case $a<a^{\prime}$. For a natural number $n$ such that $\delta\left(\alpha_{n}\right)=1$ and $A_{n-1}$ contains the inverse image of $\beta_{0}$, we see that $\alpha_{n}$ belongs to $B_{0}$. Let $I$ be the set consisting of such elements $\alpha_{n}$. We have then $B_{0}=B_{0}^{\prime} \cup I$. If $\alpha_{n}$ belongs to $I$, then $\ell\left(S^{(n)}\right)-\ell\left(S^{(n-1)}\right)=\left|A_{n-1}\right|-\left|A_{n}\right|-1$ since $\beta_{0}$ does not belongs to $A_{n}$. Thus we have $\ell\left(S^{(a)}\right)-\ell\left(S^{(0)}\right) \leq\left|A_{0}\right|-|I|$. As $\ell\left(S^{(a+m)}\right)-\ell\left(S^{(a)}\right)=$ $\left|B_{0}\right|-\left|B_{m}\right|$ with $\left|B_{m}\right|>0$, we have

$$
\ell\left(S^{\prime}\right) \leq \ell(S)-1-\left|B_{m}\right|
$$

Thus we see that $\ell\left(S^{\prime}\right)<\ell(S)-1$.

By Propositions 3.8 and 3.10 , to classify generic specializations, we may suppose that there exist non-negative integers $a$ and $b$ such that $A_{a}=\emptyset$ and $B_{b}=\emptyset$ for a small modification. For the ABS $S^{(a+b)}$, if elements $t$ and $t^{\prime}$ of $T\left(S^{(a+b)}\right)$ satisfy that $t<t^{\prime}$ and $\delta(t)=\delta\left(t^{\prime}\right)$, then $\pi(t)<\pi\left(t^{\prime}\right)$ holds. Thus in this assumption, the specialization $S^{\prime}$ of $S$ by $\left(0_{i}^{r}, 1_{j}^{q}\right)$ is obtained by $S^{\prime}=S^{(a+b)}$. We call this $\operatorname{ABS} S^{(a+b)}$ the full modification by $\left(0_{i}^{r}, 1_{j}^{q}\right)$.

\subsection{Proof of Theorem 1.5}

The main purpose of this section is to prove Theorem 1.5. The notation is as above. Let $S^{(0)}$ denote the small modification by $\left(0_{i}^{r}, 1_{j}^{q}\right)$ with $r<q$ and $0_{i}^{r}<1_{j}^{q}$ in $T(S)$. Using Lemma 3.11 and Proposition 3.12 below, we will show that, to classify generic specializations, we may suppose that the $r$-th segment of the Newton polygon $\xi$ is adjacent to the $q$-th segment, i.e., $q=r+1$, see Corollary 3.13 .

Lemma 3.11. Let $S$ be the $A B S$ associated to $N_{\xi}$ with $\xi=\sum_{i=1}^{z}\left(m_{i}, n_{i}\right)$ a Newton polygon. Let $0^{r}$ and $1^{q}$ be elements of $T(S)$ satisfying that the $r$-th segment is not adjacent to the $q$-th segment, i.e., $q>r+1$, and $0^{r}<1^{q}$ in $T(S)$. Then there exists an element $t^{x}$ of $T(S)$ such that $r<x<q$ and $0^{r}<t^{x}<1^{q}$.

Proof. For a Newton polygon $\xi$, we write $\mathrm{Q}(\xi)$ for the assertion: For elements $0^{r}$ and $1^{q}$ of the $A B S$ associated to $N_{\xi}$ satisfying that $r+1<q$ and $0^{r}<1^{q}$, there exists an element $t^{x}$ such that $r<x<q$ and $0^{r}<t^{x}<1^{q}$. It suffices to treat the case $z=3, r=1$ and $q=3$. If $\lambda_{1}=\lambda_{2}$ (resp. $\lambda_{2}=\lambda_{3}$ ) holds, then we immediately have the desired element $t^{x}$ since for elements $0_{i}^{1}<1_{j}^{3}$, the element $0_{i}^{2}$ (resp. $1_{j}^{2}$ ) satisfy $0_{i}^{1}<0_{i}^{2}<1_{j}^{3}\left(\right.$ resp. $0_{i}^{1}<1_{j}^{2}<1_{j}^{3}$ ).

From now on, we assume that the slopes are different from each other. We treat Newton polygons satisfying one of the following:
(i) $\lambda_{3}<1 / 2 \leq \lambda_{2}<\lambda_{1}$
(ii) $\lambda_{3}<\lambda_{2} \leq 1 / 2<\lambda_{1}$. 
By the duality, if $\mathrm{Q}(\xi)$ is true for all $\xi$ satisfying (i), then $\mathrm{Q}(\xi)$ holds for all $\xi$ satisfying (ii). Suppose that $\xi$ satisfies (i). Put $h_{x}=m_{x}+n_{x}$ for all $x$. By Lemma 2.17, in the subset $T\left(S_{1} \oplus S_{3}\right)$ of $T(S)$, there exists no element $t$ satisfying that $0_{n_{1}}^{1}<t<1_{1}^{3}$ or $0_{h_{1}}^{1}<t<1_{n_{3}+1}^{3}$, where the ABS $S_{i}$ corresponds to the $\mathrm{DM}_{1} N_{m_{i}, n_{i}}$. Hence it is enough to show that there exist elements $t_{x}^{2}$ and $t_{y}^{2}$ such that $0_{n_{1}}^{1}<t_{x}^{2}<1_{1}^{3}$ and $0_{h_{1}}^{1}<t_{y}^{2}<1_{n_{3}+1}^{3}$ in $T(S)$. If $\lambda_{2}$ is greater than $1 / 2$, then these elements are obtained by $t_{x}^{2}=0_{n_{2}}^{2}$ and $t_{y}^{2}=0_{h_{2}}^{2}$. In fact, by Proposition 3.5, we have $0_{n_{1}}^{1}<0_{n_{2}}^{2}$ and $0_{h_{1}}^{1}<0_{h_{2}}^{2}$. Moreover, considering the subset $T\left(S_{2} \oplus S_{3}\right)$ of $T(S)$, by Lemma 2.17, we obtain $0_{n_{2}}^{2}<1_{1}^{3}$ and $0_{h_{2}}^{2}<1_{n_{3}+1}^{3}$. If $\lambda_{2}$ equal $1 / 2$, then the desired elements $t_{x}^{2}$ and $t_{y}^{2}$ are obtained by $1_{1}^{2}$ and $0_{2}^{2}$ respectively.

Let us show that $\mathrm{Q}(\xi)$ holds for any Newton polygons $\xi$. We claim that

(A) If $\mathrm{Q}\left(\xi^{\mathrm{D}}\right)$ holds, then $\mathrm{Q}(\xi)$ also holds;

(B) If $m_{i} \leq n_{i}$ for all $i$ and $\mathrm{Q}\left(\xi^{\mathrm{C}}\right)$ holds, then $\mathrm{Q}(\xi)$ also holds.

If the claims (A) and (B) are true, then for a Newton polygon $\xi$ in NP, using the function $\Phi: \mathrm{NP} \rightarrow \mathrm{NP}^{\text {sep }}$ defined in Section 3.1, the proposition is reduced to the case (i) or (ii), and we complete the proof. The claim (A) is obvious. Let us show (B). Let $S$ (resp. $R$ ) denote the ABS associated to $\xi$ (resp. $\xi^{\mathrm{C}}$ ). By Lemma 3.3, we can regard $T(R)$ as a subset of $T(S)$ as ordered sets. Let $U$ (resp. $V)$ be the subset of $T(R) \times T(R)(\operatorname{resp} . T(S) \times T(S))$ consisting of pairs $\left(0^{1}, 1^{3}\right)$ of elements of $T(R)$ (resp. $\left.T(S)\right)$ satisfying $0^{1}<1^{3}$. Again by Lemma 3.3 , since all elements $t$ of $T(S)-T(R)$ satisfy $\Delta(S)(t)=0$, we have $U=V$. Thus (B) holds.

Compare Examples 2.14 and 2.15 for an example of Lemma 3.11

In Proposition 3.12 below, we give necessary conditions for specializations to be generic. Moreover, by Corollary 3.13 below, we see that to classify generic specializations of the ABS corresponding to a minimal $\mathrm{DM}_{1}$, it suffices to treat specializations by pairs $\left(0^{r}, 1^{r+1}\right)$.

Proposition 3.12. Let $S$ be the $A B S$ associated to $N_{\xi}$ with $\xi=\sum_{i=1}^{z}\left(m_{i}, n_{i}\right)$. For the small modification $S^{(0)}$ by $\left(0_{i}^{r}, 1_{j}^{q}\right)$, if either of the following assertions

(i) the set $A_{0}$ contains an element $0^{x}$ with $r<x$, or

(ii) the set $B_{0}$ contains an element $1^{x}$ with $x<q$

holds, then the specialization $S^{\prime}$ by $\left(0_{i}^{r}, 1_{j}^{q}\right)$ is not generic.

Proof. Put $\pi=\Pi\left(S^{(0)}\right)$ and $\delta=\Delta\left(S^{(0)}\right)$. Set $\alpha_{n}=\pi^{n}\left(0_{i}^{r}\right)$ and $\beta_{n}=\pi^{n}\left(1_{j}^{q}\right)$ for all $n$. By Propositions 3.8 and 3.10 , we may assume that there exist non-negative integers $a$ and $b$ such that $A_{a}=\emptyset$ and $B_{b}=\emptyset$. Put

$$
B_{0}^{\prime}=\left\{t \in T\left(S^{(0)}\right) \mid \beta_{0}<t \text { and } \pi(t)<\beta_{1} \text { in } S^{(0)} \text { with } \delta(t)=1\right\} .
$$


For this set, $\ell(S)-\ell\left(S^{(0)}\right)=\left|A_{0}\right|+\left|B_{0}^{\prime}\right|+1$. Define $d(n)$ by

$$
d(n)= \begin{cases}\left|A_{n}\right|-\left|A_{n+1}\right| & \text { if } n<a \\ \left|B_{n}\right|-\left|B_{n+1}\right| & \text { if } n \geq a\end{cases}
$$

We have then $\ell\left(S^{(n+1)}\right)-\ell\left(S^{(n)}\right) \leq d(n)$. Clearly $\ell\left(S^{\prime}\right)-\ell\left(S^{(0)}\right) \leq\left|A_{0}\right|+\left|B_{0}\right|$ holds. First, we show that $\ell\left(S^{\prime}\right)-\ell\left(S^{(0)}\right) \leq\left|A_{0}\right|+\left|B_{0}^{\prime}\right|$. Let $I$ be the subset of $B_{0}$ consisting of elements which are of the form $\alpha_{m}$ for some natural number $m$. We have then $\left|B_{0}\right| \leq\left|B_{0}^{\prime}\right|+|I|$. Let $m$ be a non-negative integer such that $A_{m}$ contains the inverse image of $\beta_{0}$. We have then $\ell\left(S^{(m+1)}\right)-\ell\left(S^{(m)}\right) \leq d(m)-1$ since $\beta_{0}$ is not contained in $A_{m+1}$. If $\delta\left(\alpha_{m+1}\right)=1$, then $\alpha_{m+1}$ belongs to $I$. Hence we see $\ell\left(S^{(a)}\right)-\ell\left(S^{(0)}\right) \leq\left|A_{0}\right|-|I|$. Moreover, we have $\ell\left(S^{\prime}\right)-\ell\left(S^{(a)}\right) \leq\left|B_{0}\right|$. Thus we get the desired inequality.

Let us see that in the case (i) the specialization is not generic. Let $m$ be the minimum number such that the set $A_{m}$ contains no element $t^{x}$ with $r<x$. Take an element $t^{x}$ of $A_{m-1}$ with $r<x$. Put $t=\pi\left(t^{x}\right)$. Note that since $t$ does not belong to $A_{m}$, the values of $t$ and $\alpha_{m}$ are different from each other. Now we claim that $\delta(t)=1$ and $\delta\left(\alpha_{m}\right)=0$. If $\delta(t)=0$ and $\delta\left(\alpha_{m}\right)=1$ is true, then there exists an element $1^{x}$ satisfying $\alpha_{m}<1^{x}<t$ in $T(S)$. In fact, if $1_{n}^{x}<\alpha_{m}$ holds in $T(S)$ for all $n$, then we have $1_{m_{x}}^{x}<1_{m_{r}}^{r}$ with $r<x$. By Proposition 3.5 this is a contradiction. Thus we see that the set $A_{m}$ contains the element $1^{x}$. This contradicts the minimality of $m$. Hence we have $\delta(t)=1$ and $\delta\left(\alpha_{m}\right)=0$, and it implies that $\ell\left(S^{(m)}\right)-\ell\left(S^{(m-1)}\right)<d(m)$. This induces that $\ell\left(S^{(a)}\right)-\ell\left(S^{(0)}\right)<\left|A_{0}\right|-|I|$.

Let us treat the case (ii). In the same way as the case (i), if $B_{0}$ contains an element $t^{x}$ with $x<q$, then there exists a non-negative integer $m$ such that $\ell\left(S^{(m)}\right)-\ell\left(S^{(m-1)}\right)<$ $d(m)$. In fact, for the minimum number $m$ such that $B_{m}$ contains no element $t^{x}$ with $x<q$, take an element $t^{x}$ of $B_{m-1}$ satisfying $x<q$. Then for $t=\pi\left(t^{x}\right)$, we have $\delta(t)=0$ and $\delta\left(\beta_{m}\right)=1$ since if $\delta(t)=1$ and $\delta\left(\beta_{m}\right)=0$ is true, then there exists an element $0^{x}$ of $T(S)$ satisfying that $t<0^{x}<\beta_{m}$ by Proposition 3.5. This implies that $B_{m}$ contains an element $0^{x}$, and this contradicts the minimality of $m$.

By the above, in the cases (i) and (ii), we have $\ell\left(S^{\prime}\right)-\ell\left(S^{(0)}\right)<\left|A_{0}\right|+\left|B_{0}^{\prime}\right|$, and by $\ell(S)-\ell\left(S^{(0)}\right)=\left|A_{0}\right|+\left|B_{0}^{\prime}\right|+1$, it follows that $\ell\left(S^{\prime}\right)<\ell(S)-1$.

Corollary 3.13. If the $r$-the segment is not adjacent to the $q$-th segment, i.e., $q>r+1$, then the specialization is not generic.

Proof. Put $\delta=\Delta(S)$. For a small modification by $\left(0^{r}, 1^{q}\right)$, it follows from Lemma 3.11 that there exists an element $t^{x}$ of $T(S)$ such that $0^{r}<t^{x}<1^{q}$ and $r<x<q$. If $\delta\left(t^{x}\right)=0$, then the element $t^{x}$ belongs to $A_{0}$, and the assertion immediately follows from Proposition 3.12, Let us see the case $\delta\left(t^{x}\right)=1$. If the set $B_{0}$ contains $t^{x}$, then by Proposition 3.12 we complete the proof. Let us consider the case that the element $t^{x}$ with 
$\delta\left(t^{x}\right)=1$ does not belong to $B_{0}$. For the set $B_{0}^{\prime}$ of Proposition $3.12, t^{x}$ is contained in $B_{0}^{\prime}$. Thus, if $t^{x}$ does not belong to $B_{0}$, then the minimum number $a$ satisfying $A_{a}=\emptyset$ must satisfy that $a \geq a^{\prime}$, where $a^{\prime}$ is the minimum number such that $\alpha_{a^{\prime}}=\beta_{0}$ since $\left|B_{0}\right|<\left|B_{0}^{\prime}\right|$ only if $a \geq a^{\prime}$. In this case, we have $\left|B_{0}\right|<\left|B_{0}^{\prime}\right|+|I|$, where the set $I$ is the same as the set in the proof of Proposition 3.12. Clearly we have $\ell(S)-\ell\left(S^{(0)}\right)=\left|A_{0}\right|+\left|B_{0}^{\prime}\right|+1$. As $\ell\left(S^{\prime}\right)-\ell\left(S^{(0)}\right)<\left|A_{0}\right|+\left|B_{0}\right|-|I|$, we are done.

Example 3.14 below is an example of a specialization which is not generic. This example indicates that for the small modification $S^{(0)}$ by $\left(0^{r}, 1^{q}\right)$, if the $r$-th segment is not adjacent to the $q$-the segment, then the specialization by $0^{r}$ and $1^{q}$ is not generic. Compare with Example 2.20.

Example 3.14. For the ABS $S$ of $\xi=(2,7)+(1,2)+(3,5)$, consider the small modification by $\left(0_{4}^{1}, 1_{2}^{3}\right)$. Then the ABS $S^{(0)}$ is

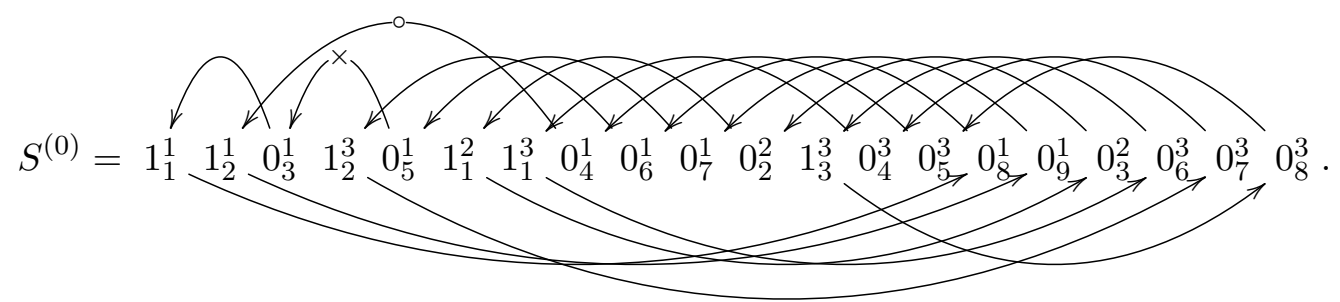

We have $A_{0}=\left\{0_{5}^{1}\right\}$ and $A_{1}=\emptyset$. Thus we see $a=1$. We have sets $B_{0}=\left\{1_{1}^{2}, 1_{1}^{3}\right\}$ and $B_{1}=\left\{0_{3}^{2}, 0_{6}^{3}\right\}$ with the ABS $S^{(2)}$

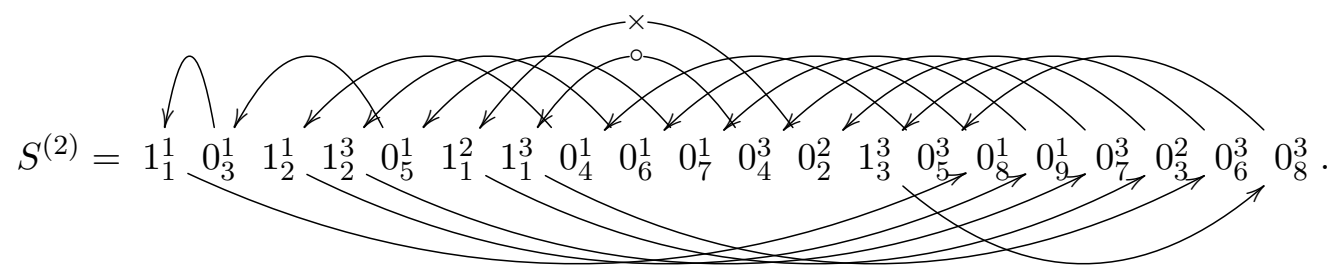

By the ABS $S^{(2)}$, we obtain the set $B_{2}=\left\{0_{2}^{2}\right\}$. Similarly, we obtain $B_{3}=\left\{1_{1}^{2}\right\}, B_{4}=\left\{0_{3}^{2}\right\}$ and $B_{5}=\emptyset$ with the ABS's $S^{(4)}, S^{(5)}$ and $S^{(6)}$. Hence we have $b=5$, and the full modification $S^{(6)}$ is equal to $S^{\prime}$ of Example 2.15 .

Let $\xi=\sum_{i=1}^{z}\left(m_{i}, n_{i}\right)$ be a Newton polygon. Let $S$ be the ABS of the $\mathrm{DM}_{1} N_{\xi}$. Recall that the ABS $S$ is the direct sum $S=\bigoplus_{i=1}^{z} S_{i}$ of ABS's $S_{i}$ corresponding to the $\mathrm{DM}_{1} N_{m_{i}, n_{i}}$. Propositions 3.8 and 3.10 imply that all generic specializations are given by full modifications. Now let us show Theorem 1.5. This theorem says that to determine generic specializations of $H(\xi)$ it is enough to deal with Newton polygons consisting of two segments. 
Proof of Theorem 1.5. Let $S$ be the ABS of $N_{\xi}$. Let us construct a bijection map

$$
\bigsqcup_{i=1}^{z-1}\left\{\text { generic specializations of } R_{i}\right\} \longrightarrow\{\text { generic specializations of } S\},
$$

where $R_{i}$ denotes the ABS corresponding to the $\mathrm{DM}_{1}$ of the two slopes Newton polygon $\left(m_{i}, n_{i}\right)+\left(m_{i+1}, n_{i+1}\right)$. As we can regard $T\left(R_{r}\right)$ as a subset of $T(S)$ as ordered sets, we denote by $t_{i}^{r}$ (resp. $t_{j}^{r+1}$ ) the $i$-th (resp. the $j$-th) element of the first (resp. the second) component of $R_{r}$, corresponding to the segment $\left(m_{r}, n_{r}\right)$ (resp. $\left(m_{r+1}, n_{r+1}\right)$ ). By Corollary 3.13 it suffices to show the claim: The full modification of $S$ by $\left(0_{i}^{r}, 1_{j}^{r+1}\right)$ is generic if and only if the full modification of $R_{r}$ by $\left(0_{i}^{r}, 1_{j}^{r+1}\right)$ is generic. For a small modification $S^{(0)}$ by $\left(0_{i}^{r}, 1_{j}^{r+1}\right)$ of $S$, we use the same notation as Definitions 2.18 and 2.19. To simplify, set $\pi=\Pi\left(S^{(0)}\right)$ and $\delta=\Delta\left(S^{(0)}\right)$. Let $E$ (resp. $F$ ) denote the subset of $A_{0}$ (resp. $\left.B_{0}\right)$ consisting of elements $0^{x}$ (resp. $1^{y}$ ) with $x \neq r$ (resp. $y \neq r+1$ ). Put $R=S_{r} \oplus S_{r+1}$. Let $R^{\prime}$ be a generic specialization of $R$ by $\left(0_{i}^{r}, 1_{j}^{r+1}\right)$, and let $S^{\prime}$ be the specialization of $S$ by $\left(0_{i}^{r}, 1_{j}^{r+1}\right)$. For the small modifications $S^{(0)}$ and $R^{(0)}$ by $\left(0_{i}^{r}, 1_{j}^{r+1}\right)$, we clearly have $\ell(S)-\ell\left(S^{(0)}\right)=\ell(R)-\ell\left(R^{(0)}\right)+|E|+|F|$. To show the claim, it suffices to see that $\ell(S)-\ell\left(S^{\prime}\right)=\ell(R)-\ell\left(R^{\prime}\right)$. So we will show that

$$
\ell\left(S^{\prime}\right)-\ell\left(S^{(0)}\right)=\ell\left(R^{\prime}\right)-\ell\left(R^{(0)}\right)+|E|+|F|
$$

Let $m$ be the minimum number such that $\alpha_{m}=1_{m_{r}}^{r}$. Let $C_{0}, C_{1}, \ldots$ be the $A$-sequence associated to $R, 0_{i}^{r}$ and $1_{j}^{r+1}$. Note that as $R^{\prime}$ is generic, there exists a non-negative integer $a^{\prime}$ such that $C_{a^{\prime}}$ is empty. We show the following three claims:

(a) for every element $0^{x}$ of $E$, we have $x<r$,

(b) there exists no element $t^{x}$ of $A_{m}$ such that $x \neq r$,

(c) there exists a non-negative integer $a$ satisfying $A_{a}=\emptyset$, and

$$
\ell\left(S^{(a)}\right)-\ell\left(S^{(0)}\right)=\ell\left(R^{\left(a^{\prime}\right)}\right)-\ell\left(R^{(0)}\right)+|E| .
$$

Put $q=r+1$. To show (a), suppose $r<x$. For an element $0^{x}$ of $A_{0}$, we have $0^{x}<0_{i}^{r}$ in $T\left(S^{(0)}\right)$. So it is clear that $0^{x}<1_{j}^{q}$ in $T(S)$. Then clearly $0_{m_{x}+1}^{x}<0_{m_{q}+1}^{q}$ in $T(S)$, and by Proposition 3.5 we have $x<q$. As $r$ and $q$ are adjacent, this is a contradiction. We have shown (a). To show (b), suppose that $A_{m-1}$ contains an element $t^{x}$ with $x \neq r$. Then there exists an element $t_{0}^{x}$ of $A_{0}$ such that $\pi^{m-1}\left(t_{0}^{x}\right)=t^{x}$ by Proposition 3.6, and by (a), we see $x<r$. Then $\delta\left(\pi\left(t^{x}\right)\right)=0$. In fact, if $\delta\left(\pi\left(t^{x}\right)\right)=1$, then we have $1_{m_{r}}^{r}<\pi\left(t^{x}\right) \leq 1_{m_{x}}^{x}$ with $x<r$. By Proposition 3.5, this is a contradiction. Since $\delta\left(\alpha_{m}\right)=1$, we obtain (b). Let us show (c). Note that the sets $C_{n}$ satisfy $A_{n}=C_{n} \cup\left\{t^{x} \in A_{n} \mid x \neq r\right\}$ for 
all $n$. Thus, using (b), there exists a non-negative integer $a$ such that $A_{a}$ is empty. Let $n$ be a non-negative integer. Suppose that the set $A_{n}$ contains an element $t^{x}$ such that $\pi\left(t^{x}\right)$ does not belong to $A_{n+1}$. Note that $\delta\left(\alpha_{n+1}\right)$ and $\delta\left(\pi\left(t^{x}\right)\right)$ are different from each other by the definition of $A_{n+1}$. To show (3.6), it suffices to show that $\delta\left(\alpha_{n+1}\right)=1$ and $\delta\left(\pi\left(t^{x}\right)\right)=0$. In fact, let $E_{n}$ be the subset of $A_{n}$ consisting of elements $t^{x}$ with $x \neq r$ satisfying that $\pi\left(t^{x}\right)$ does not belong to $A_{n+1}$. If $\delta\left(\alpha_{n+1}\right)=1$ and $\delta\left(\pi\left(t^{x}\right)\right)=0$ are true, then $\ell\left(S^{(n+1)}\right)-\ell\left(S^{(n)}\right)=\ell\left(R^{(n+1)}\right)-\ell\left(R^{(n)}\right)+\left|E_{n}\right|$, and by (b), we get the equation (3.6). To read a contradiction, suppose that $\delta\left(\alpha_{n+1}\right)=0$ and $\delta\left(\pi\left(t^{x}\right)\right)=1$. We have then $0_{m_{r}+1}^{r} \leq \alpha_{n+1}<\pi\left(t^{x}\right)<0_{m_{x}+1}^{x}$. As $x<r$ holds by (a), this contradicts Proposition 3.5.

Similarly, we have the "dual" of (a), (b) and (c). Let $m^{\prime}$ be the minimum number such that $\beta_{m^{\prime}}=0_{m_{q}+1}^{q}$. Then

(d) for every element $1^{y}$ of $F$, we have $q<y$,

(e) there exists no element $t^{y}$ of $B_{m^{\prime}}$ such that $y \neq q$,

(f) we have

$$
\ell\left(S^{\prime}\right)-\ell\left(S^{(a)}\right)=\ell\left(R^{\prime}\right)-\ell\left(R^{\left(a^{\prime}\right)}\right)+|F|
$$

By (3.6) and (3.7), we get 3.5). Thus, for a generic specialization $R^{\prime}$ obtained by $\left(0_{i}^{r}, 1_{j}^{r+1}\right)$, the specialization $S^{\prime}$ of $S$ obtained by $\left(0_{i}^{r}, 1_{j}^{r+1}\right)$ is generic. In the same way, one can check if a specialization $S^{\prime}$ of $S$ by $\left(0_{i}^{r}, 1_{j}^{r+1}\right)$ is generic, then the specialization $R^{\prime}$ of $R$ by $\left(0_{i}^{r}, 1_{j}^{r+1}\right)$ is also generic.

Example 3.15. Let $\xi=(2,5)+(3,7)+(3,2)$. For the small modification obtained by $\left(0_{5}^{2}, 1_{1}^{3}\right)$, the set $A_{0}$ contains $0_{5}^{1}$. One can check that the specialization by $\left(0_{5}^{2}, 1_{1}^{3}\right)$ is generic. Moreover, for the ABS corresponding to $N_{(3,7)+(3,2)}$, the specialization by $\left(0_{5}^{1}, 1_{1}^{2}\right)$ is also generic.

\section{The case of Newton polygons consisting of two slopes}

In this section, to solve Problem 1.4, we give a proof of Theorem 1.6. In Section 3, we have seen that it suffices to deal with Newton polygons consisting of two segments to classify generic specializations. Thus we mainly treat Newton polygons consisting of two segments in this section. The notation is as Sections 2 and 3 .

For a Newton polygon $\xi$, we have a bijection from $B(\xi)$ to $B\left(\xi^{\mathrm{D}}\right)$, see the paragraph after Theorem 1.6. See 1.2 for the definition of $B(\xi)$. Moreover, to get a bijection between $B(\xi)$ and $B\left(\xi^{\mathrm{C}}\right)$, we use Lemma 3.3 . If Theorem 1.6 is true, then using the two 
bijections and the map $\Phi: \mathrm{NP} \rightarrow \mathrm{NP}^{\text {sep }}$ defined in Section 3.1, we can reduce Problem 1.4 into the case of $[3]$.

Proof of Theorem 1.6. Let $S$ (resp. $R$ ) denote the ABS associated to $\xi$ (resp. $\xi^{C}$ ). Let $G(S)$ (resp. $G(R)$ ) denote the set of generic specializations of $S$ (resp. $R$ ). The assertion is paraphrased as follows: There exists a map $G(S) \rightarrow G(R)$ defined by sending a generic specialization $S^{\prime}$ of $S$ obtained by $\left(0_{i}^{1}, 1_{j}^{2}\right)$ to the generic specialization $R^{\prime}$ of $R$ obtained by $\left(0_{i}^{1}, 1_{j}^{2}\right)$, and this map is bijective. The set $T(R)$ can be regarded as a subset of $T(S)$ as ordered sets, see Lemma 3.3. Again by Lemma 3.3, we have

$$
\begin{aligned}
& \left\{\left(0^{1}, 1^{2}\right) \in T(S) \times T(S) \mid 0^{1}<1^{2} \text { in } T(S)\right\} \\
= & \left\{\left(0^{1}, 1^{2}\right) \in T(R) \times T(R) \mid 0^{1}<1^{2} \text { in } T(R)\right\} .
\end{aligned}
$$

Take elements $0_{i}^{1}$ and $1_{j}^{2}$, with $0_{i}^{1}<1_{j}^{2}$, of $T(S)$ such that the specialization $S^{\prime}$ of $S$ by $\left(0_{i}^{1}, 1_{j}^{2}\right)$ is generic. Let $\left\{A_{n}\right\}$ (resp. $\left.\left\{A_{n}^{\prime}\right\}\right)$ be the $A$-sequence associated to $S, 0_{i}^{1}$ and $1_{j}^{2}$ (resp. $R, 0_{i}^{1}$ and $1_{j}^{2}$ ), see Definition 2.18 for the definition of the $A$-sequence. Clearly we have $A_{0}=A_{0}^{\prime}$ and $\ell\left(S^{(0)}\right)=\ell\left(R^{(0)}\right)$. For a non-negative integer $n$, suppose that $A_{n}=A_{n}^{\prime}$ and $\ell\left(S^{(n)}\right)=\ell\left(R^{(n)}\right)$. If elements $t$ of $A_{n}^{\prime}$ satisfy that $\Delta(R)(t)=0$, then by Lemma 3.3 we see that $A_{n+1}=A_{n+1}^{\prime}$ and $\ell\left(S^{(n+1)}\right)=\ell\left(R^{(n+1)}\right)$. Moreover, if elements $t$ of $A_{n}^{\prime}$ satisfy $\Delta(R)(t)=1$, then it follows from Lemma 3.3 that $A_{n+2}=A_{n+1}^{\prime}$ and $\ell\left(S^{(n+2)}\right)=\ell\left(R^{(n+1)}\right)$. Note that in this case $A_{n+1}$ is not empty. Thus, as the specialization $S^{\prime}$ of $S$ by $\left(0_{i}^{1}, 1_{j}^{2}\right)$ is generic, it follows from Proposition 3.8 and the above that there exists the minimum number $a\left(\right.$ resp. $\left.a^{\prime}\right)$ such that $A_{a}$ (resp. $\left.A_{a^{\prime}}\right)$ is empty. Similarly, for the $B$-sequence $\left\{B_{n}\right\}$ (resp. $\left\{B_{n}^{\prime}\right\}$ ) associated to $S, 0_{i}^{1}$ and $1_{j}^{2}$ (resp. $R, 0_{i}^{1}$ and $1_{j}^{2}$ ), we have $B_{0}=B_{0}^{\prime}$ and $\ell\left(S^{(a)}\right)=\ell\left(R^{\left(a^{\prime}\right)}\right)$. See Definition 2.19 for the definition of the $B$-sequence. For a non-negative integer $n$, suppose that $B_{n}=B_{n}^{\prime}$. Similarly as above, we have

$$
\begin{cases}B_{n+1}=B_{n+1}^{\prime} \text { and } \ell\left(S^{(a+n+1)}\right)=\ell\left(R^{\left(a^{\prime}+n+1\right)}\right) & \text { if } \Delta(R)(t)=0 \text { for } t \in B_{n}^{\prime}, \\ B_{n+2}=B_{n+1}^{\prime} \text { and } \ell\left(S^{(a+n+2)}\right)=\ell\left(R^{\left(a^{\prime}+n+1\right)}\right) & \text { otherwise. }\end{cases}
$$

Thus we see that $\ell\left(R^{\prime}\right)=\ell(R)-1$ if and only if $\ell\left(S^{\prime}\right)=\ell(S)-1$.

The following is an example of Theorem 1.6. See also Example 2.20.

Example 4.1. Let $\xi=(2,7)+(3,5)$. We have then $\xi^{\mathrm{C}}=(2,5)+(3,2)$. Let $R$ be the ABS associated to $N_{\xi^{\mathrm{C}}}$. For this ABS

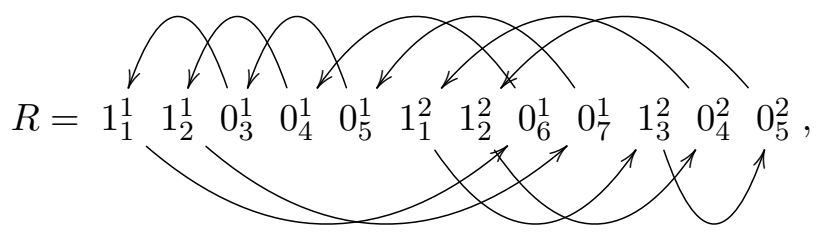

the specialization $R^{\prime}$ obtained by exchanging $0_{4}^{1}$ and $1_{2}^{2}$ is 


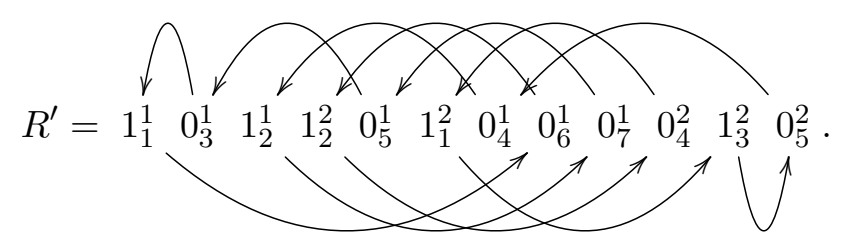

For the small modification by $\left(0_{4}^{1}, 1_{2}^{2}\right)$, we have sets $A_{0}=\left\{0_{5}^{1}\right\}, A_{1}=\emptyset, B_{0}=\left\{1_{1}^{2}\right\}$ and $B_{1}=\emptyset$. Thus we have $a=1$ and $b=1$. Compare with Example 2.20 $A$-sequences and $B$-sequences of $S$ and $R$. By Example 2.14, we can regard $T(R)$ as a subset of $T(S)$ as ordered sets. One can check that these $R$ and $R^{\prime}$ satisfy $\ell\left(R^{\prime}\right)=\ell(R)-1$. Moreover, the sets of pairs $\left(0_{i}^{1}, 1_{j}^{2}\right)$ constructing generic specializations for ABS's corresponding to $N_{\xi}$ and $N_{\xi^{\mathrm{C}}}$ are both

$$
\left\{\left(0_{4}^{1}, 1_{1}^{2}\right),\left(0_{4}^{1}, 1_{2}^{2}\right),\left(0_{5}^{1}, 1_{1}^{2}\right),\left(0_{5}^{1}, 1_{2}^{2}\right),\left(0_{6}^{1}, 1_{3}^{2}\right),\left(0_{7}^{1}, 1_{3}^{2}\right)\right\}
$$

5. Determining Newton polygons of generic specializations

In this section, we prove Theorem 1.8. Let $\xi=\sum_{i}\left(m_{i}, n_{i}\right)$ be a Newton polygon. By Corollary 3.13 , for the ABS corresponding to $N_{\xi}$, generic specializations are obtained by specializations by $0^{r}$ and $1^{r+1}$. First, we see that it suffices to deal with $\xi$ consisting of two segments in order to show Theorem 1.8 .

Lemma 5.1. If Theorem 1.8 is true for $\xi$ with two segments, then Theorem 1.8 is true for any $\xi$.

Proof. Let $w \in B(\xi)$. Then there exists the generic specialization $w^{\prime}$ in $B\left(\xi_{i}\right)$ corresponding to $w$ via the map obtained in Theorem 1.5 for some $i$ with $\xi_{i}=\left(m_{i}, n_{i}\right)+\left(m_{i+1}, n_{i+1}\right)$. By hypothesis, we have a Newton polygon $\zeta^{\prime}$ satisfying (i) and (ii) of Theorem 1.8 for $\xi_{i}$ and $w^{\prime}$. Put $\zeta=\left(m_{1}, n_{1}\right)+\cdots+\left(m_{i-1}, n_{i-1}\right)+\zeta^{\prime}+\left(m_{i+2}, n_{i+2}\right)+\cdots+\left(m_{z}, n_{z}\right)$. Then this $\zeta$ satisfies (i) and (ii) of Theorem 1.8 for $\xi$ and $w$.

The key statement for the proof of Theorem 1.8 is Theorem 5.2 below. We show the theorem by reducing it to the case that the Newton polygon of a minimal $\mathrm{DM}_{1}$ consists of two segments, where one slope is less than $1 / 2$ and the other slope is greater than $1 / 2$, see [3, Section 5] for the proof in the case.

Theorem 5.2. Let $\xi=\left(m_{1}, n_{1}\right)+\left(m_{2}, n_{2}\right)$ be a Newton polygon consisting of two segments. Assume $m_{2} n_{1}-m_{1} n_{2}>1$. For any element $w$ of $B(\xi)$, there exist a specialization $w^{-}$of $w$ and a segment $\rho$ such that

$$
w^{-}=w^{\prime} \oplus w_{\rho}
$$

with $w^{\prime} \in B\left(\xi^{\prime}\right)$ and $\rho=(c, d)$, where $\xi^{\prime}$ is either $\left(m_{1}-c, n_{1}-d\right)+\left(m_{2}, n_{2}\right)$ or $\left(m_{1}, n_{1}\right)+$ $\left(m_{2}-c, n_{2}-d\right)$ : 

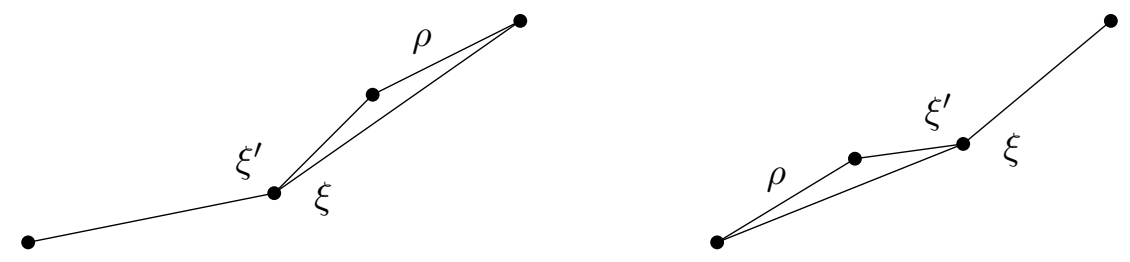

so that the area of the region surrounded by $\xi, \xi^{\prime}$ and $\rho$ is one.

Proof. If $\xi=\left(m_{1}, n_{1}\right)+\left(m_{2}, n_{2}\right)$ belongs to NPsep, then we have shown this fact in 3 , Proposition 5.1]. In this case, it is straightforward to see that $m_{2} n_{1}-m_{1} n_{2}=1$ if and only if $\xi=(0,1)+(1,0)$.

Let us show the statement by induction on the height of $\xi$. Consider the case that $\xi$ does not belong to $\mathrm{NP}^{\mathrm{sep}}$. Then $\xi$ satisfies $m_{i} \leq n_{i}$ for $i=1,2$ or $n_{i} \leq m_{i}$ for $i=1,2$. If the Newton polygon $\xi$ satisfies the latter, then we replace $\xi$ with $\xi^{\mathrm{D}}=$ $\left(n_{2}, m_{2}\right)+\left(n_{1}, m_{1}\right)$. Thus it suffices to see the case that $\xi$ satisfies the former. Recall that for a Newton polygon $\xi=\left(m_{1}, n_{1}\right)+\left(m_{2}, n_{2}\right)$ satisfying $m_{i} \leq n_{i}$ for $i=1,2$, we set $\xi^{\mathrm{C}}=\left(m_{1}, n_{1}-m_{1}\right)+\left(m_{2}, n_{2}-m_{2}\right)$, see the first paragraph of Section 3.1. Put $\eta=\xi^{\mathrm{C}}$. Let $w \in B(\xi)$. We denote by $w^{\mathrm{C}}$ the image of the map $B(\xi) \rightarrow B(\eta)$ of Theorem 1.6 . It is clear that the height of $\eta$ is less than that of $\xi$. By the hypothesis of induction, there exist a specialization $\left(w^{\mathrm{C}}\right)^{-}$of $w^{\mathrm{C}}$ and a segment $\tau$ such that $\left(w^{\mathrm{C}}\right)^{-}=v \oplus w_{\tau}$, with $v \in B\left(\eta^{\prime}\right)$, where $\eta^{\prime}$ is uniquely determined by $\eta$ and $\tau$ so that the area of the region surrounded by $\eta, \eta^{\prime}$ and $\tau$ is one. Then we construct required $w^{-}$as follows. We denote by $S$ (resp. $R$ ) the ABS corresponding to $w$ (resp. $w^{\mathrm{C}}$ ), which is the type of $N_{\xi}^{-}$ (resp. $N_{\xi^{\mathrm{C}}}^{-}$) in the notation of 3 , Section 5]. By Theorem 1.6 if we write $\left\{s_{1}<\cdots<s_{h}\right\}$ (resp. $\left.\left\{s_{1}^{\prime}<\cdots<s_{h^{\prime}}^{\prime}\right\}\right)$ for the ordered set $T(S)$ (resp. $\left.T(R)\right)$, with $h=m_{1}+n_{1}+m_{2}+n_{2}$ and $h^{\prime}=n_{1}+n_{2}$, then $s_{x}=s_{x}^{\prime}$ for $x=1, \ldots, h^{\prime}$. Moreover, if $\Delta(S)\left(s_{x}\right)=1$ for an integer $x$, then $\Pi(S)^{2}\left(s_{x}\right)=\Pi(R)\left(s_{x}^{\prime}\right)$, see Theorem 1.6. Suppose that the specialization $R^{-}$of $R$ by $\left(0_{i}^{1}, 1_{j}^{2}\right)$ corresponds to $\left(w^{\mathrm{C}}\right)^{-}=v \oplus w_{\tau}$. Let us consider the specialization $S^{-}$of $S$ by $\left(0_{i}^{1}, 1_{j}^{2}\right)$. If we denote by $\left\{t_{1}<^{\prime} \cdots<^{\prime} t_{h}\right\}$ and $\left\{t_{1}^{\prime}<^{\prime} \cdots<^{\prime} t_{h^{\prime}}^{\prime}\right\}$ the ordered sets $T\left(S^{-}\right)$and $T\left(R^{-}\right)$respectively, then $t_{x}=t_{x}^{\prime}$ for $x=1, \ldots, h^{\prime}$. Moreover, if $\Delta\left(S^{-}\right)\left(t_{x}\right)=1$ for an integer $x$, then $\Pi\left(S^{-}\right)^{2}\left(t_{x}\right)=\Pi\left(R^{-}\right)\left(t_{x}^{\prime}\right)$. Thus we see that $S^{-}$corresponding to $w^{-}=w^{\prime} \oplus w_{\rho}$, with $\left(w^{\prime}\right)^{\mathrm{C}}=v$ and $\rho^{\mathrm{C}}=\tau$.

Remark 5.3. Let $\xi=\left(m_{1}, n_{1}\right)+\left(m_{2}, n_{2}\right)$ be a Newton polygon consisting of two segments. If $m_{2} n_{1}-m_{1} n_{2}=1$, then $B(\xi)$ consists of only one element, and it is $w_{\zeta}$ with $\zeta=$ $\left(m_{1}+m_{2}, n_{1}+n_{2}\right)$. Note that $\zeta \prec \xi$ is saturated. This fact is proved by reducing the problem to the case of $\xi=(0,1)+(1,0)$ via the map $\Phi: \mathrm{NP} \rightarrow \mathrm{NP}^{\mathrm{sep}}$, in the similar way as the proof of Theorem 5.2 .

Theorem 1.8 follows from Theorem 5.2 . Indeed, this can be proven in the same way as the proof of [3. Theorem 1.3], but we write the proof for the reader's convenience. 
Proof of Theorem 1.8. Thanks to Lemma 5.1, we may assume that $\xi$ consists of two segments. Put $\xi=\left(m_{1}, n_{1}\right)+\left(m_{2}, n_{2}\right)$. Let us show the statement by induction on the value $m_{2} n_{1}-m_{1} n_{2}$. The minimum value is one. We have discussed the case of the minimal value in Remark 5.3 .

Suppose that $m_{2} n_{1}-m_{1} n_{2}$ is greater than one. Then, by Theorem 5.2 , for $w \in B(\xi)$, there exist a specialization $w^{-}$and a segment $\rho$ such that $w^{-}=w^{\prime} \oplus w_{\rho}$, with $w^{\prime} \in B\left(\xi^{\prime}\right)$, where $\xi^{\prime}=\left(m_{1}^{\prime}, n_{1}^{\prime}\right)+\left(m_{2}^{\prime}, n_{2}^{\prime}\right)$ is uniquely determined by $\xi$ and $\rho$ so that the area of the region surrounded by $\xi, \xi^{\prime}$ and $\rho$ is one. Note that $m_{2}^{\prime} n_{1}^{\prime}-m_{1}^{\prime} n_{2}^{\prime}$ is less than $m_{2} n_{1}-m_{1} n_{2}$. By the hypothesis of induction, there exists a Newton polygon $\zeta^{\prime}$ such that $\zeta^{\prime} \prec \xi^{\prime}$ is saturated, and $w_{\zeta^{\prime}} \subset w^{\prime}$. Then the Newton polygon $\zeta=\zeta^{\prime}+\rho$ satisfies that $\zeta \prec \xi$ is saturated, and $w_{\zeta} \subset w$.

Remark 5.4. Thanks to Theorems 5.2 and 1.8 , by the same argument as the proof of 4 , Theorem 5.1], for a Newton polygon $\xi$ and for a generic specialization $w$ of $w_{\xi}$, we obtain a sequence

$$
w_{\zeta}=w^{(n)} \subset w^{(n-1)} \subset \cdots \subset w^{(1)} \subset w^{(0)}=w,
$$

where $w^{(i+1)}$ is a specialization of $w^{(i)}$, and $w^{(i)}$ corresponds to $N_{\xi^{i}}^{-} \oplus\left(N_{\rho} \oplus N_{\rho^{1}} \oplus \cdots \oplus\right.$ $\left.N_{\rho^{i-1}}\right)$ in the notation of 3 , Section 5], where $\rho^{i}$ is the $\rho$ appearing when we apply 3 , Proposition 5.1] to $\xi^{i}$. Moreover, $n+1$ is equal to twice the area of the region surrounded by $\zeta$ and $\xi$ (cf. [4, Section 4]). Note that $\zeta \prec \xi$ is saturated. From this, for $w$ and $w^{-}$of Theorem 5.2, we obtain $\ell\left(w^{-}\right)=\ell(w)-1$, i.e., $w^{-}$is a generic specialization of $w$ because otherwise (5.1) implies $\ell\left(w_{\xi}\right)-\ell\left(w_{\zeta}\right)<n+1$, which contradicts [4, Corollary 5.2].

Here is an example of Theorems 5.2 and 1.8 .

Example 5.5. Let $\xi=(2,7)+(3,5)$. Let $R$ denote the ABS corresponding to $\xi^{\mathrm{C}}=$ $(2,5)+(3,2)$. Let $R^{-}$be the generic specialization obtained by exchanging $0_{4}^{1}$ and $1_{2}^{2}$. Consider the full modification by $\left(0_{3}^{1}, 1_{2}^{1}\right)$ for $R^{-}$. Then this full modification can be described as

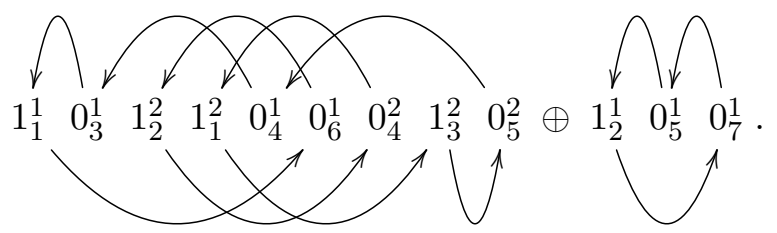

The former component is a generic specialization of $N_{(1,3)+(3,2)}$, and the latter component is associated to the Newton polygon $(1,2)$ consisting of one segment. For the first component, let us consider the full modification by $\left(0_{4}^{2}, 1_{3}^{2}\right)$. We have then the specialization $N_{(1,3)+(2,1)}^{-} \oplus N_{(1,1)}$. One can see that we obtain the Newton polygon $\zeta^{\prime}$ satisfying (i) and (ii) of Theorem 1.8 for $\xi^{\mathrm{C}}$ by $\zeta^{\prime}=2(1,2)+3(1,1)$. 
Next, for the ABS $S$ corresponding to $N_{\xi}$, let $S^{-}$be the generic specialization obtained by exchanging $0_{4}^{1}$ and $1_{2}^{2}$. The specialization of $S^{-}$by $0_{3}^{1}$ and $1_{2}^{1}$ is described as

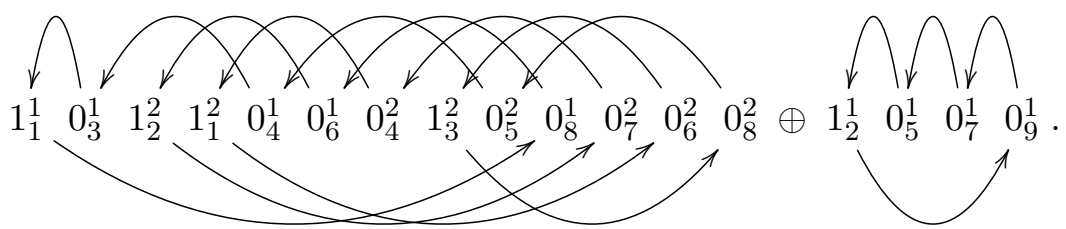

The former component is a generic specialization of $N_{(1,4)+(3,5)}$, and the latter component is associated to the segment $(1,3)$. For the first component, let us consider the full modification by $\left(0_{4}^{2}, 1_{3}^{2}\right)$. We have then the specialization $N_{(1,4)+(2,3)}^{-} \oplus N_{(1,2)}$. If we write $\zeta$ for the Newton polygon satisfying (i) and (ii) of Theorem 1.8 for $\xi$, we have then $\zeta=2(1,3)+3(1,2)$, and one can easily check that $\zeta^{\mathrm{C}}=\zeta^{\prime}$.

\section{Acknowledgments}

I thank the referee for careful reading and helpful comments. This paper is written when the author was a Ph.D. student. I thank the supervisor Professor Harashita for the constant support from the early stage of this paper. This research is partially supported by Grant-in-Aid for JSPS Fellows.

\section{References}

[1] N. Bourbaki, Lie Groups and Lie Algebras, Chapters 4-6, Translated from the 1968 French original by Andrew Pressley, Elements of Mathematics (Berlin), SpringerVerlag, Berlin, 2002.

[2] S. Harashita, Configuration of the central streams in the moduli of abelian varieties, Asian J. Math. 13 (2009), no. 2, 215-250.

[3] N. Higuchi, On the boundary components of central streams in the two slopes case, Kodai Math. J. 43 (2020), no. 2, 366-408.

[4] N. Higuchi and S. Harashita, On specializations of minimal p-divisible groups, Yokohama Math. J. 64 (2018), 1-20.

[5] A. J. de Jong, Crystalline Dieudonné module theory via formal and rigid geometry, Inst. Hautes Études Sci. Publ. Math. No. 82 (1995), 5-96 (1996).

[6] N. M. Katz, Slope filtration of F-crystals, in: Journées de Géométrie Algébrique de Rennes (Rennes, 1978), Vol. I, pp. 113-163, Astérisque, 63, Soc. Math. France, Paris, 1979. 
[7] H. Kraft, Kommutative algebraische p-Gruppen, Sonderforschungsbereich. Bonn, September 1975. Ms. 86 pp.

[8] B. Moonen, A dimension formula for Ekedahl-Oort strata, Ann. Inst. Fourier (Grenoble) 54 (2004), no. 3, 666-698.

[9] B. Moonen and T. Wedhorn, Discrete invariants of varieties in positive characteristic, Int. Math. Res. Not. 2004 (2004), no. 72, 3855-3903.

[10] P. Norman and F. Oort, Moduli of abelian varieties, Ann. of Math. (2) 112 (1980), no. $3,413-439$.

[11] F. Oort, A stratification of a moduli space of abelian varieties, in: Moduli of Abelian Varieties (Texel Island, 1999), 345-416, Progr. Math. 195, Birkhäuser, Basel, 2001.

[12] Foliations in moduli spaces of abelian varieties, J. Amer. Math. Soc. 17 (2004), no. 2, 267-296.

[13] Minimal p-divisible groups, Ann. of Math. (2) 161 (2005), no. 2, 1021-1036.

[14] E. Viehmann and T. Wedhorn, Ekedahl-Oort and Newton strata for Shimura varieties of PEL type, Math. Ann. 356 (2013), no. 4, 1493-1550.

[15] T. Wedhorn, The dimension of Oort strata of Shimura varieties of PEL-type, in: Moduli of Abelian Varieties (Texel Island, 1999), 441-471, Progr. Math. 195, Birkhäuser, Basel, 2001.

Nobuhiro Higuchi

Graduate School of Environment and Information Sciences, Yokohama National

University, 79-1 Tokiwadai, Hodogaya-ku, Yokohama 240-8501, Japan

E-mail address: tachyondr107@gmail.com 